\title{
Bandwidth of graphs resulting from the edge clique covering problem
}

\author{
Konrad Engel \\ Institut für Mathematik \\ Universität Rostock \\ D-18051 Rostock, Germany \\ konrad.engel@uni-rostock.de
}

\author{
Sebastian Hanisch * \\ Institut für Mathematik \\ Universität Rostock \\ D-18051 Rostock, Germany \\ sebastian.hanisch@uni-rostock.de
}

Submitted: Mar 17, 2017; Accepted: Nov 1, 2018; Published: Dec 21, 2018

(C) The authors. Released under the CC BY-ND license (International 4.0).

\begin{abstract}
Let $n, k, b$ be integers with $1 \leqslant k-1 \leqslant b \leqslant n$ and let $G_{n, k, b}$ be the graph whose vertices are the $k$-element subsets $X$ of $\{0, \ldots, n\}$ with $\max (X)-\min (X) \leqslant b$ and where two such vertices $X, Y$ are joined by an edge if $\max (X \cup Y)-\min (X \cup Y) \leqslant b$. These graphs are generated by applying a transformation to maximal $k$-uniform hypergraphs of bandwidth $b$ that is used to reduce the (weak) edge clique covering problem to a vertex clique covering problem. The bandwidth of $G_{n, k, b}$ is thus the largest possible bandwidth of any transformed $k$-uniform hypergraph of bandwidth $b$. For $b \geqslant \frac{n+k-1}{2}$, the exact bandwidth of these graphs is determined. Moreover, the bandwidth is determined asymptotically for $b=o(n)$ and for $b$ growing linearly in $n$ with a factor $\beta \in(0,1]$, where for one case only bounds could be found. It is conjectured that the upper bound of this open case is the right asymptotic value.
\end{abstract}

Mathematics Subject Classifications: 05C78, 05C69, 05C85

\section{Introduction}

The bandwidth problem for graphs is to find a labelling of the vertices with different integers, such that the maximum absolut value of the difference of the labels of two adjacent vertices is minimal. There are many applications such as efficient storage of sparsely populated symmetric matrices, which arise e.g. from discretization of partial differential equations, cf. [21]. Several other applications, including the placement problem for modules of a VLSI design, the binary constraint satisfaction problem and the minimization of effects of noise in the multichannel communication of data are discussed

*Supported by DFG graduate school $1505 / 2$ welisa. 
e.g. in $[3,5,8]$. The bandwidth problem was shown to be NP-hard [19] and even an approximation with a ratio better than 2 is NP-hard [10], so several heuristics such as the Cuthill-McKee-Algorithm [9] or some similar approaches, cf. [12], are very popular in applications. However, for some graph classes the exact bandwidth is known. These include the path, the cycle, the complete graph, the complete bipartite graph [6], the hypercube [14], the grid graph [7], special Hamming graphs [17] and several others, cf. [5]. However, there are still many graphs, where the exact bandwidth is unknown, such as the general Hamming graphs, cf. $[2,16]$. In this paper, we consider graphs $G_{n, k, b}, 1 \leqslant k-1 \leqslant b \leqslant n$, whose vertices are those $k$-element subsets of $\{0, \ldots, n\}$, for which the difference of the maximum and the minimum is at most $b$. There is an edge between two vertices if the difference of the maximum and the minimum of the union of the corresponding sets is at most $b$. We start by introducing the necessary notation and a motivation in Section 2 and study some basic properties in Section 3. Based on that, we determine the exact bandwidth for these graphs in the case of $b \geqslant \frac{n+k-1}{2}$ in Section 4 . In Section 5, we present some asymptotic results for $n \rightarrow \infty$ in the case of $b=o(n)$. The results of Sections 4 and 5 are summarized by the following theorem:

Theorem 1. Let $k$ be a fixed positive integer and $1 \leqslant k-1 \leqslant b \leqslant n$.

a) If $b \geqslant \frac{n+k-1}{2}$, then

$$
B\left(G_{n, k, b}\right)=\left\lceil\frac{(n+1)\left(\begin{array}{c}
b \\
k-1
\end{array}\right)-(k-1)\left(\begin{array}{c}
b+1 \\
k
\end{array}\right)+\left(\begin{array}{c}
2 b-n+1 \\
k
\end{array}\right)-2}{2}\right\rceil .
$$

b) If $b=o\left(n^{\frac{1}{k+1}}\right)$, then for sufficiently large $n$

$$
B\left(G_{n, k, b}\right)=k\left(\begin{array}{l}
b \\
k
\end{array}\right) .
$$

If $b=o(n)$, then

$$
B\left(G_{n, k, b}\right) \sim k\left(\begin{array}{l}
b \\
k
\end{array}\right) \text { as } n \rightarrow \infty .
$$

Sections 6 to 10 discuss the case $b \sim \beta n$ with $\beta \in(0,1]$. Of course, in view of Theorem 1 a) it would be enough to consider only $\beta \leqslant \frac{1}{2}$. But the main results given by the next theorem are also true for $\beta>\frac{1}{2}$.

Theorem 2. Let $k \geqslant 2$ be a fixed positive integer, but $n \rightarrow \infty$. Let $b \sim \beta n$ and let $1=q \beta+r$, where $q$ is a positive integer and $0 \leqslant r<\beta$. Let

$$
\begin{aligned}
& c_{1}(\beta, k)=\frac{\beta^{k}}{k !}\left(k-\frac{k-1}{q}\right), \\
& c_{2}(\beta, k)=\frac{\beta^{k-1}}{(q+1) k !}(k-(k-1) \beta), \\
& c_{3}(\beta, k)=\frac{(\beta-r)^{k}}{(q+1) k !} q^{k-1} .
\end{aligned}
$$


a) If $r \leqslant \frac{q-1}{q^{2}+q-1}$, then $B\left(G_{n, k, b}\right) \sim c_{1}(\beta, k) n^{k}$.

b) If $r>\frac{q-1}{q^{2}+q-1}$, then $\max \left\{c_{1}(\beta, k), c_{2}(\beta, k)+\frac{1}{q^{k-1}} c_{3}(\beta, k)\right\} n^{k} \lesssim B\left(G_{n, k, b}\right) \lesssim\left(c_{2}(\beta, k)+\right.$ $\left.c_{3}(\beta, k)\right) n^{k}$.

The part b) gives only bounds instead of an exact asymptotic value if $q>1$, i.e., $\beta \leqslant \frac{1}{2}$. We strongly conjecture that the RHS bound is the right value. The bounds are not too far away from each other because

$$
\frac{c_{2}(\beta, k)}{c_{3}(\beta, k)}=\left(\frac{\beta}{q \beta-q r}\right)^{k} \frac{q}{\beta}(k-(k-1) \beta) \geqslant\left(\frac{\beta}{q \beta-q r}\right)^{k} \frac{k(q-1)+1}{\beta} \geqslant 6
$$

since $\beta>q \beta-q r$ iff $r>\frac{q-1}{q^{2}+q-1}$ and $k \geqslant 2, q \geqslant 2, \beta \leqslant \frac{1}{2}$.

Let $U=\left\{\beta \in\left(0, \frac{1}{2}\right]: r>\frac{q-1}{q^{2}+q-1}\right\}$ be the set of numbers $\beta$ for which part b) applies and thus the exact asymptotic value is still unknown. Note that $r>\frac{q-1}{q^{2}+q-1}$ iff $\frac{1}{q+1}<\beta<$ $\frac{q}{q^{2}+q-1}$. Thus the Lebesgue measure of $U$ is equal to $\sum_{q=2}^{\infty}\left(\frac{q}{q^{2}+q-1}-\frac{1}{q+1}\right)=0.119 \ldots$, i.e., for the "majority" of numbers $\beta \in\left(0, \frac{1}{2}\right]$ the exact value is known.

The corresponding asymptotically optimal labellings change dynamically with $\beta$ in a nontrivial way. In Section 10, this is described in detail. This unexpected behavior makes the study interesting and challenging.

The proof of Theorem 2 is based on a reduction to a continuous problem on the unit square $[0,1]^{2}$. Riemann integrals and elementary geometric arguments suffice. The embedding into a more difficult continuous problem on the unit cube was used by Harper [15] to obtain bounds for the bandwidth of Hamming graphs. Also for the edge-bandwidth of multidimensional grids and Hamming graphs (the bandwidth of the line graph of these graphs) Harper's reduction to the unit cube was applied in [1]. Asymptotic bounds for the bandwidth of the $d$-ary de Bruijn graph were obtained in [20] by an approach based on the use of a continuous domain.

\section{Notation and motivation}

Let $[n]=\{1, \ldots, n\}$ and $[i, j]=\{i, i+1, \ldots, j-1, j\}$ with $i, j \in \mathbb{Z}, i \leqslant j$. In particular, $[0, n]=\{0,1, \ldots, n\}$. For a graph $G=(V, E)$ with $|V|=n$ vertices, a proper numbering of $G$ is a bijection $f: V \rightarrow[n]$. For two vertices $u, v \in V$, we call $d_{f}(u, v)=\mid f(u)-$ $f(v) \mid$ the $f$-distance of $u$ and $v$. Let $f$ be a proper numbering of a graph $G$. The bandwidth of $f$, denoted $B_{f}(G)$, is given by $B_{f}(G)=\max \{|f(u)-f(v)|:\{u, v\} \in E\}$, i.e., the maximal $f$-distance. The bandwidth of $G$ is defined by $B(G)=\min \left\{B_{f}(G)\right.$ : $f$ is a proper numbering of $G\}$. A bandwidth numbering of $G$ is a numbering $f$ such that $B(G)=B_{f}(G)$. This definition can be easily generalized to hypergraphs $H=(V, E)$. There we have $B_{f}(H)=\max \{|f(u)-f(v)|: \exists e \in E$ with $u, v \in e\}$.

Now we formally define the subject of our study. Let $k$ and $b$ be positive integers with $b \geqslant k-1$. For $A \subseteq[0, n]$ let $\underline{A}=\min (A)$ and $\bar{A}=\max (A)$. Further let $\left(\begin{array}{c}{[0, n]} \\ k\end{array}\right)=\{X \subseteq$ 
$[0, n]:|X|=k\}$. Then $G_{n, k, b}$ is the graph with vertex set

$$
V_{n, k, b}=\left\{X \in\left(\begin{array}{c}
{[0, n]} \\
k
\end{array}\right): \bar{X}-\underline{X} \leqslant b\right\}
$$

and edge set

$$
E_{n, k, b}=\left\{\{X, Y\} \in V_{n, k, b}: \overline{X \cup Y}-\underline{X \cup Y} \leqslant b\right\} .
$$

These graphs arise in the following context: Let $G=(V, E)$ be a graph. A clique is a subset of $V$ that induces a complete subgraph of $G$. Consider the following transformation, which was used in the NP-completeness proof of the edge clique covering problem in [18] by reducing it to the vertex clique covering problem. Let $V=[n]$. Then $\tilde{G}=(\tilde{V}, \tilde{E})$ is constructed by setting $\tilde{V}=E$ and $\left\{\{i, j\},\left\{i^{\prime}, j^{\prime}\right\}\right\} \in \tilde{E}$ if there is a clique $C \subseteq V$ with $i, j, i^{\prime}, j^{\prime} \in C$. Let $\bar{\chi}_{v}(G)$ (resp. $\bar{\chi}_{e}(G)$ ) be the vertex clique covering number (resp. edge clique covering number) of the graph $G=(V, E)$, i.e., the minimal number of cliques whose induced subgraphs cover all vertices (resp. edges) of $G$. It can be shown, that $\bar{\chi}_{e}(G)=\bar{\chi}_{v}(\tilde{G})$, which is the essential part of the NP-completeness proof for the edge clique covering problem, since the transformation can be done in polynomial time. Here we want to generalize this result for hypergraphs $H=(V, E)$. We consider the 2-section graph of $H$, i.e., the graph $G_{H}=\left(V, E_{H}\right)$ on the vertex set of $H$ where $\{u, v\} \in E_{H}$ if there is an edge of $H$ containing $u$ and $v$. A subset $C$ of $V$ is called a weak clique of $H$ if $C$ is a clique in $G_{H}$. A weak edge clique covering of $H$ is a family $\mathcal{C}$ of weak cliques of $H$ such that for all $e \in E$ there is some $C \in \mathcal{C}$ with $e \subseteq C$. The weak edge clique covering number of $H$ is the smallest size $\bar{\chi}_{e}(H)$ of a weak edge clique covering of $H$. It will turn out that the computation of $\bar{\chi}_{e}(H)$ can be simplified by the following transformation. The weak edge clique graph of $H$ is the graph $\tilde{G}_{H}=(\tilde{V}, \tilde{E})$ where $\tilde{V}=E$ and two vertices of $\tilde{G}_{H}$, i.e., edges $e, e^{\prime}$ of $H$, are adjacent if there is a weak clique $C$ of $H$ containing $e$ and $e^{\prime}$ as subsets. With these definitions we prove the following proposition.

Proposition 3. Let $H=(V, E)$ be a hypergraph. Then

$$
\bar{\chi}_{e}(H)=\bar{\chi}_{v}\left(\tilde{G}_{H}\right) .
$$

Proof. Let $\mathcal{C}$ be a weak edge clique covering of $H$ of size $\bar{\chi}_{e}(H)$. For each $C \in \mathcal{C}$ let $\tilde{C}=\{e \in E: e \subseteq C\}$. Then $\tilde{C}$ is a clique in $\tilde{G}_{H}$ and $\tilde{\mathcal{C}}=\{\tilde{C}: C \in \mathcal{C}\}$ is a vertex clique covering of $\tilde{G}_{H}$. Consequently $\bar{\chi}_{e}(H) \geqslant \bar{\chi}_{v}\left(\tilde{G}_{H}\right)$. Now let $\tilde{\mathcal{C}}$ be a vertex clique covering of $\tilde{G}_{H}$ of size $\bar{\chi}_{v}\left(\tilde{G}_{H}\right)$. For each $\tilde{C} \in \mathcal{C}$ let $C=\bigcup_{e \in \tilde{C}} e$. Then $C$ is a weak clique in $H$. To verify this fact, we pick two arbitrary vertices $x, y \in C$ and show that they are adjacent in $G_{H}$. First we consider the case that there is an edge $e \in \tilde{C}$ with $x, y \in e$. Then, by construction of $G_{H},\{x, y\} \in E_{H}$. The alternative is, that there are 2 edges $e, e^{\prime} \in \tilde{C}$ with $x \in e$ and $y \in e^{\prime}$. Since $e$ and $e^{\prime}$ are adjacent in $\tilde{G}_{H}$ there is a weak clique of $H$ containing both edges, which implies the adjacency of $x$ and $y$. Moreover, $\tilde{\mathcal{C}}$ is a weak edge clique covering of $H$. Consequently, $\bar{\chi}_{v}\left(\tilde{G}_{H}\right) \geqslant \bar{\chi}_{e}(H)$.

Thus, from an algorithmic point of view, it is enough to study the vertex clique covering problem. For bounded bandwidth, and more generally for bounded treewidth, there is 
a linear time dynamic programming algorithm for the solution [4]. In an application, which will be described below, we were lead to the weak edge covering problem on a hypergraph whose bandwidth is small (and thus, theoretically, considered as bounded). This implies the following question: Given a hypergraph $H$ of bandwidth $b$, how large can be the bandwidth of the weak edge clique graph $\tilde{G}_{H}$ of $H$ ? Here we discuss only $k$-uniform hypergraphs though many results can be simply generalized to hypergraphs whose edges have size at most $k$. For later computations, it is more suitable to take $[0, n]$ as the vertex set of $H$ instead of $[1, n]$. If, without loss of generality, $f(i)=i$ is the bandwidth numbering of $H$, then, obviously, $\tilde{G}_{H}$ has maximal bandwidth if $H$ contains all $k$-element subsets $X$ of $[0, n]$ with $\bar{X}-\underline{X} \leqslant b$. In this case, $\tilde{G}_{H}$ is exactly the graph $G_{n, k, b}$, which motivates the study of $G_{n, k, b}$.

We came to these questions in the study of multielectrode recordings of neuronal signals, so-called spikes. Such recordings are carried out on multielectrode arrays, which can be used in-vivo or in-vitro. The denser the electrodes are placed the more likely it is for the neurons to be simultaneously recorded at different electrodes. The resulting similarities in the recordings of the electrodes can provide useful information. In [11] we developed an algorithm to estimate the (unknown) neighborhood of a neuron, i.e., the set of electrodes which record the signals of this neuron. Such neighborhood information is also used as an additional tool in [22] for the so called spike sorting, which is an estimated assignment of the recorded signals to the neurons.

Fix a short time interval in which several electrodes record signals. We consider these electrodes as vertices of a graph, which we call similarity-graph for the fixed time interval. First we mention that some neurons may always spike simultaneously. We combine such a set of neurons to one (artificial) new neuron. It might be an accident that two electrodes record a signal at almost the same time, but the simultaneous recording can also be caused by the fact that one spiking neuron has contact to both electrodes. Thus we do not test only one short time interval but several such intervals. If there are sufficiently many simultaneous recordings of two (or $k$ ) fixed electrodes, one may expect that these recordings are indeed caused by only one neuron and thus we draw an edge (hyperedge) between the corresponding vertices in the similarity-graph.

By algorithmic reasons, it is easier to check only pairs of electrodes, see [11]. But, with some more effort, also $k$-element subsets of electrodes could be checked for similarities if $k$ is small. This leads to edges and hyperedges of electrodes. If a spiking neuron has contact to an unknown set $S$ of electrodes, all edges between any two vertices of $S$ (all hyperedges of any $k$ vertices of $S$ ) are drawn in the similarity-graph. Though these edges may also be caused by different simultaneously spiking neurons having contact in each case to two (or $k$ ) neurons, it is more likely that only one neuron is the source. Such a neuron yields the edges of a weak clique in the similarity-graph. Once the similarity-graph is constructed, it remains the question what is the basic cause for this graph. A reasonable answer is that as few as possible neurons yield the graph. Consequently, a minimum weak edge clique covering has to be determined. Because of the bounded length of the axons, only nearby electrodes, which are placed in form of a two-dimensional bounded grid (or some similar variants), may have contact to the same neuron. Hence the similarity-graph is a relatively 
sparse graph and edges are only drawn between electrodes which have a small Euclidean distance. Thus it is reasonable to expect that also this graph has a small bandwidth.

For more information and more detailed explanations, we refer to the dissertation of the second author [13].

\section{Some basic properties}

Let $k-1 \leqslant b^{\prime} \leqslant b$. It is easy to see that $G_{n, k, b^{\prime}}$ is a subgraph of $G_{n, k, b}$. For asymptotic estimates, we need the auxiliary graph $G_{n, k, b^{\prime}, b}$ that is defined as the subgraph of $G_{n, k, b}$ induced by the vertex set of $G_{n, k, b^{\prime}}$, i.e., the vertices are the $k$-element subsets $X$ of $[0, n]$ with $\bar{X}-\underline{X} \leqslant b^{\prime}$ and $\{X, Y\}$ is an edge iff $\overline{X \cup Y}-\underline{X \cup Y} \leqslant b$. Note that $G_{n, k, b, b}=G_{n, k, b}$. From the definition of the bandwidth it follows immediately that

$$
B\left(G_{n, k, b^{\prime}}\right) \leqslant B\left(G_{n, k, b}\right) \text { and } B\left(G_{n, k, b^{\prime}, b}\right) \leqslant B\left(G_{n, k, b}\right) .
$$

Lemma 4. Let $X$ and $Y$ be two distinct vertices of $G_{n, k, b^{\prime}, b}$. They are adjacent iff $\bar{X}-\underline{Y} \leqslant$ $b$ and $\bar{Y}-\underline{X} \leqslant b$.

Proof. Let $X$ and $Y$ be adjacent. Then $\bar{X}-\underline{Y} \leqslant \overline{X \cup Y}-\underline{X \cup Y} \leqslant b$ and, analogously, $\bar{Y}-\underline{X} \leqslant b$.

Now let $\bar{X}-\underline{Y} \leqslant b$ and $\bar{Y}-\underline{X} \leqslant b$. Then $\overline{X \cup Y}-\underline{X \cup Y}=\max \{\bar{X}, \bar{Y}\}-\min \{\underline{X}, \underline{Y}\}=$ $\max \{\bar{X}-\underline{X}, \bar{X}-\underline{Y}, \bar{Y}-\underline{X}, \bar{Y}-\underline{Y}\} \leqslant b$.

Note that $[i, i+k-1] \in V_{n, k, b}$ iff $0 \leqslant i \leqslant n-(k-1)$. Moreover, for $0 \leqslant i<j \leqslant n-(k-1)$, the vertices $[i, i+k-1]$ and $[j, j+k-1]$ are adjacent iff $j \leqslant i+b-(k-1)$.

Lemma 5. Let $0 \leqslant i<j \leqslant n-(k-1)$. The vertices $[i, i+k-1]$ and $[j, j+k-1]$ have distance $\left\lceil\frac{j-i}{b-k+1}\right\rceil$ in $G_{n, k, b^{\prime}, b}$.

Proof. Let $j-i=q(b-k+1)+r$ where $q$ is an integer and $1 \leqslant r \leqslant b-k+1$. Then $\left\lceil\frac{j-i}{b-k+1}\right\rceil=q+1$. Obviously, the vertices $[i, i+(k-1)],[i+b-(k-1), i+b],[i+2 b-$ $2(k-1), i+2 b-(k-1)], \ldots,[i+q b-q(k-1), i+q b-(q-1)(k-1)],[j, j+(k-1)]$ form a path in $G_{n, k, b^{\prime}, b}$ of length $q+1$. Thus the distance is at most $q+1$.

If the vertices $X_{0}=[i, i+(k-1)], X_{1}, \ldots, X_{l-1},[j, j+(k-1)]=X_{l}$ form any path of length $l$ in $G_{n, k, b^{\prime}, b}$, then, for $t=1, \ldots, l, \bar{X}_{t}-\underline{X}_{t-1} \leqslant b$ and $\bar{X}_{t}-\underline{X}_{t} \geqslant k-1$, which implies

$$
\underline{X}_{t}-\underline{X}_{t-1} \leqslant b-(k-1) .
$$

Summing up the inequalities (2) for $t=1, \ldots, l$ yields $j-i \leqslant l(b-k+1)$. Since $j-i>q(b-k+1)$ we have $l \geqslant q+1$ and thus the distance is at least $q+1$.

Lemma 6. Let $X, Y$ be two vertices of $G_{n, k, b^{\prime}, b}$ and let $\underline{X}<\underline{Y}$ or $\underline{X}=\underline{Y}$ as well as $\bar{X}<\bar{Y}$. Then $X$ and $Y$ have distance at most $\left\lceil\frac{\bar{Y}-\underline{X}-b}{b-k+1}\right\rceil+1$. 
Proof. Let $i=\underline{X}$ and $j=\bar{Y}$.

Case 1. $j-i \leqslant b$. Then $\bar{Y}-\underline{X} \leqslant b$ and $\bar{X}-\underline{Y} \leqslant \bar{X}-\underline{X} \leqslant b^{\prime} \leqslant b$. Consequently, $X$ and $Y$ are adjacent by Lemma 4 and their distance is 1 . Indeed, from the conditions on $X$ and $Y$ it follows that $\bar{Y}-\underline{X}>k-1$ and thus $\bar{Y}-\underline{X}-b>(-1)(b-k+1)$, which implies $\left\lceil\frac{\bar{Y}-\underline{X}-b}{b-k+1}\right\rceil+1=1$.

Case 2. $j-i>b$. Obviously, $X$ is adjacent to $X_{1}=[i+b-k+1, i+b]$ and $Y$ is adjacent to $Y_{1}=[j-b, j-b+k-1]$.

Case 2.1. $i+b-k+1 \geqslant j-b$. Then $X_{1}$ and $Y$ are adjacent since $\bar{X}_{1}-\underline{Y}=i+b-\underline{Y}=$ $\underline{X}-\underline{Y}+b \leqslant b$ and $\bar{Y}-\underline{X}_{1}=j-i-b+k-1 \leqslant b$. Thus $X$ and $Y$ have distance at most 2. Indeed, $2 \leqslant\left\lceil\frac{\bar{Y}-X-b}{b-k+1}\right\rceil+1 \leqslant\left\lceil\frac{b+(b-k+1)-b}{b-k+1}\right\rceil+1=2$.

Case 2.2. $i+b-k+1<j-b$. By Lemma $5, X_{1}$ and $Y_{1}$ have distance $\left\lceil\frac{j-b-i-(b-k+1)}{b-k+1}\right\rceil=$ $\left\lceil\frac{\bar{Y}-\underline{X}-b}{b-\bar{k}+1}\right\rceil-1$, and thus $X$ and $Y$ have distance at most $\left\lceil\frac{\bar{Y}-\underline{X}-b}{b-\overline{k+1}}\right\rceil+1$.

Lemma 7. Let $k-1 \leqslant b^{\prime} \leqslant b \sim \beta n, \delta>0$ and let $X, Y$ be two vertices of $G_{n, k, b^{\prime}, b}$ with $\bar{Y}-\underline{X} \lesssim i(1-\delta) \beta n$ as well as $\bar{X}-\underline{Y} \lesssim i(1-\delta) \beta n$, where $n \rightarrow \infty$ and $i$ is a positive integer. If $n$ is sufficiently large, then $X$ and $Y$ have distance at most $i$.

Proof. Without loss of generality, let $(\underline{X}, \bar{X}) \leqslant l e x(\underline{Y}, \bar{Y})$, i.e., $\underline{X}<\underline{Y}$ or $\underline{X}=\underline{Y}$ as well as $\bar{X}<\bar{Y}$. By Lemma $6, X$ and $Y$ have distance at most $\left\lceil\frac{i(1-\delta) \beta n-b}{b-k+1}\right\rceil+1=$ $\left\lceil\frac{i(1-\delta) \beta-\beta+o(1)}{\beta+o(1)}\right\rceil+1=\lceil i(1-\delta)-1+o(1)\rceil+1 \leqslant i$.

Lemma 8. The graph $G_{n, k, b}$ has the following number of vertices:

$$
\left|V_{n, k, b}\right|=(n-b+1)\left(\begin{array}{c}
b \\
k-1
\end{array}\right)+\left(\begin{array}{l}
b \\
k
\end{array}\right)=(n+1)\left(\begin{array}{c}
b \\
k-1
\end{array}\right)-(k-1)\left(\begin{array}{c}
b+1 \\
k
\end{array}\right) .
$$

Proof. The proof follows directly from the partition

$$
V_{n, k, b}=\bigcup_{i=0}^{n-b}\left\{X \in\left(\begin{array}{c}
{[i, i+b]} \\
k
\end{array}\right): \underline{X}=i\right\} \cup\left\{X \in\left(\begin{array}{c}
{[n-b+1, n]} \\
k
\end{array}\right)\right\} .
$$

\section{Bandwidth for $b \geqslant \frac{n+k-1}{2}$}

In the following, we often write the elements of $V_{n, k, b}$ as $k$-tuples in ascending order, i.e., $X=\left(i_{1}, \ldots, i_{k}\right)$ with $i_{1}<\cdots<i_{k}$. Then $\underline{X}=i_{1}$ and $\bar{X}=i_{k}$. Furthermore, let $\overleftarrow{X}=\left(i_{k}, \ldots, i_{1}\right)$ as well as $X^{c}=\left(n-i_{k}, \ldots, n-i_{1}\right)$. We collect all vertices that are adjacent to all other vertices in the set

$$
C=\left\{X \in V_{n, k, b}: n-b \leqslant \underline{X} \leqslant \bar{X} \leqslant b\right\} .
$$

Note that $C \neq \varnothing$ iff $b \geqslant \frac{n+k-1}{2}$ and that

$$
|C|=\left(\begin{array}{c}
2 b-n+1 \\
k
\end{array}\right)
$$


We denote the set of remaining vertices by $R=V_{n, k, b} \backslash C$ and split it into two parts:

$$
\begin{aligned}
& R^{\prime}=\{X \in R: \underline{X}+\bar{X} \neq n\}, \\
& R^{\prime \prime}=\{X \in R: \underline{X}+\bar{X}=n\} .
\end{aligned}
$$

Let $R^{\prime \prime}=R_{0}^{\prime \prime} \cup R_{1}^{\prime \prime}$ be a partition of $R^{\prime \prime}$ such that

$$
|| R_{0}^{\prime \prime}|-| R_{1}^{\prime \prime}|| \leqslant 1 .
$$

We call such partitions balanced. We define a partition $R^{\prime}=R_{0}^{\prime} \cup R_{1}^{\prime}$ of $R^{\prime}$ by

$$
\begin{aligned}
& R_{0}^{\prime}=\left\{X \in R^{\prime}: \underline{X}+\bar{X}<n\right\}, \\
& R_{1}^{\prime}=\left\{X \in R^{\prime}: \underline{X}+\bar{X}>n\right\}
\end{aligned}
$$

and with $R_{0}=R_{0}^{\prime} \cup R_{0}^{\prime \prime}$ and $R_{1}=R_{1}^{\prime} \cup R_{1}^{\prime \prime}$ we have a partition $R=R_{0} \bullet R_{1}$.

Lemma 9. We have ||$R_{0}|-| R_{1}|| \leqslant 1$.

Proof. A bijection between $R_{0}^{\prime}$ and $R_{1}^{\prime}$ is given by $X \mapsto X^{c}$. Hence we have $\left|R_{0}^{\prime}\right|=\left|R_{1}^{\prime}\right|$ and with (4) we obtain the assertion.

Recall the definition of the lexicographic ordering $<_{\text {lex }}$ on the set of all $k$-tuples of integers:

$$
\left(x_{1}, x_{2}, \ldots, x_{k}\right)<_{\text {lex }}\left(y_{1}, y_{2}, \ldots, y_{k}\right) \text { if } \exists i \in[k]\left(\forall j \in[i-1]: x_{j}=y_{j}\right) \wedge x_{i}<y_{i} .
$$

We define a proper numbering of $V_{n, k, b}$ in the form of a total order $\leqslant$. The minimal element gets label 1 , the next elements get labels $2,3, \ldots$ and the maximal element gets label $\left|V_{n, k, b}\right|$. Each total order will be given in the form of an ordinal sum of suborders: If $V_{n, k, b}=S_{1} \bullet \cdots \bullet S_{l}$ and $\leqslant_{i}$ is a total order on $S_{i}, i=1, \ldots, l$, then $V_{n, k, b}=S_{1} \oplus \cdots \oplus S_{l}$ means that the elements of $V_{n, k, b}$ are totally ordered as follows: $X \leqslant Y$ if there is some $i$ with $X, Y \in S_{i}$ and $X \leqslant_{i} Y$ or there are some $i, j$ with $i<j$ and $X \in S_{i}$ and $Y \in S_{j}$. Now we consider an arbitrary balanced partition $R^{\prime \prime}=R_{0}^{\prime \prime} \bullet R_{1}^{\prime \prime}$. We have $V_{n, k, b}=R_{0} \bullet C \sqcup R_{1}$, i.e., $l=3$ with $S_{1}=R_{0}, S_{2}=C$ and $S_{3}=R_{1}$. We define a total order $\leqslant_{\text {spo }}$, which we call the simple palindrom ordering (SPO), as follows:

$$
V_{n, k, b}=R_{0} \oplus C \oplus R_{1}
$$

with the following suborders:

1. $\forall X, Y \in R_{0}=R_{0}^{\prime} \cup R_{0}^{\prime \prime}: X \leqslant_{\text {spo }} Y$, if $X \leqslant_{\text {lex }} Y$.

2. $\forall X, Y \in C: X \leqslant_{\text {spo }} Y$, if $X \leqslant_{\text {lex }} Y$.

3. $\forall X, Y \in R_{1}=R_{1}^{\prime} \cup R_{1}^{\prime \prime}: X \leqslant_{\text {spo }} Y$, if $\overleftarrow{X} \leqslant_{\text {lex }} \overleftarrow{Y}$

Note that $\leqslant_{\text {spo }}$ depends on the particular balanced partition $R^{\prime \prime}=R_{0}^{\prime \prime} \cup R_{1}^{\prime \prime}$. Let $f_{\text {spo }}(X)$ be the label of $X \in V_{n, k, b}$ in the numbering given by the SPO. Recall that the $f_{\text {spo-distance }}$ of $X, Y \in V_{n, k, b}$ is given by

$$
d_{f_{s p o}}(X, Y)=\left|f_{\text {spo }}(X)-f_{\text {spo }}(Y)\right| .
$$


Lemma 10. Let $X, Y$ be two adjacent elements of $V_{n, k, b}$ with $X<_{\text {spo }} Y$ and maximal

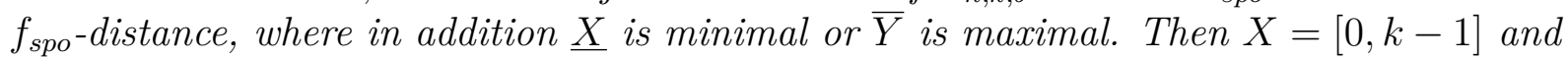
$Y=[b-k+1, b]$ or $X=[n-b, n-b+k-1]$ and $Y=[n-k+1, n]$.

Proof. If $Y \in C$, then $X=[0, k-1]$ and $Y=[b-k+1, b]$ have maximal $f_{\text {spo-distance. }}$ Analogously, if $X \in C$, then $X=[n-b, n-b+k-1]$ and $Y=[n-k+1, n]$ have maximal

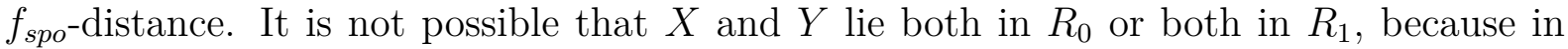
these cases $Y$ could be replaced by $[b-k+1, b]$ and $X$ by $[n-b, n-b+k-1]$, respectively. Thus it remains the case that $X \in R_{0}$ and $Y \in R_{1}$. To reach a maximal $f_{\text {spo-distance, }}$ the form $X=(\underline{X}, \underline{X}+1, \ldots, \underline{X}+k-1)$ and $Y=(\bar{Y}-k+1, \bar{Y}-k+2, \ldots, \bar{Y})$ with $\bar{Y}-\underline{X}=b$ is necessary. So we have $X=[i, i+k-1]$ and $Y=[i+b-k+1, i+b]$ for an $i \in[0, n-b]$. To prove the assertion, it is sufficient to show the following:

a) For $1 \leqslant i \leqslant \frac{n-b}{2}$, we have $d_{\text {spo }}([i-1, i+k-2],[i+b-k, i+b-1]) \geqslant d_{\text {spo }}([i, i+k-$ 1], $[i+b-k+1, i+b])$.

b) For $\frac{n-b}{2}<i \leqslant n-b$, we have $d_{\text {spo }}([i-1, i+k-2],[i+b-k, i+b-1]) \leqslant$ $d_{\text {spo }}([i, i+k-1],[i+b-k+1, i+b])$.

We note that b) follows from a) by using the bijection $X \mapsto X^{c}$ and applying the same arguments to the particular SPO $\leqslant_{s p o^{c}}$ which is given by the balanced partition $R^{\prime \prime}=$ $\left\{X^{c}: X \in R_{1}^{\prime \prime}\right\} \cup\left\{X^{c}: X \in R_{0}^{\prime \prime}\right\}$. This is possible due to

$$
X \leqslant_{\text {spo }} X^{\prime} \Longleftrightarrow X^{c} \leqslant_{\text {spoc }} X^{c} .
$$

To show a) we define $I_{i}=\left\{X \in V_{n, k, b}:[i, i+k-1] \leqslant\right.$ spo $\left.X \leqslant{ }_{\text {spo }}[i+b-k+1, i+b]\right\}$. For the proof of the inequality it is enough to show that the mapping $X=\left(i_{1}, i_{2}, \ldots, i_{k}\right) \mapsto$ $\left(i_{1}-1, i_{2}-1, \ldots, i_{k}-1\right)=\tilde{X}$ is an injection $\phi$ from $I_{i}$ to $I_{i-1}$. The injectivity is clear. Thus it remains to verify that $\tilde{X}=\phi(X) \in I_{i-1}$ if $X \in I_{i}$.

Case 1. $\tilde{X} \in C$.

This case is easy, because $C \subseteq I_{j}$ for all $j$.

Case 2. $\tilde{X} \in R_{0}$.

Case 2.1. $X \in R_{0}$. Then $[i, i+k-1] \leqslant_{l e x} X$ and thus $[i-1, i+k-2] \leqslant$ lex $\tilde{X}$, which yields $\tilde{X} \in I_{i-1}$.

Case 2.2. $X \in C$. Then $i \leqslant \frac{n-b}{2}<n-b \leqslant \underline{X}$ and thus $i-1<\underline{X}-1=\underline{\tilde{X}}$. This implies that $[i-1, i+k-2]<_{\text {lex }} \tilde{X}$, which yields $\tilde{X} \in I_{i-1}$.

Case 2.3. $X \in R_{1}$. Then $\bar{X} \leqslant \overline{[i+b-k+1, i+b]}=i+b$ because of $X \leqslant$ spo $[i+b-$ $k+1, i+b]$ and due to $\underline{X}+\bar{X} \geqslant n$ we have $\underline{X} \geqslant n-i-b$. This implies $\underline{\tilde{X}}=\underline{X}-1 \geqslant$ $n-i-b-1 \geqslant i-1$ because of $i \leqslant \frac{n-b}{2}$. Hence $\tilde{X} \in I_{i-1}$.

Case 3. $\tilde{X} \in R_{1}$.

Case 3.1. $X \in R_{0}$. This is not possible because $\underline{\tilde{X}}+\overline{\tilde{X}}=\underline{X}+\bar{X}-2<n$, which contradicts $\tilde{X} \in R_{1}$.

Case 3.2. $X \in C$. Then $\bar{X} \leqslant b$, which implies $\bar{X} \leqslant b-1 \leqslant i+b-2<\overline{[i+b-k, i+b-1]}$ due to $i \geqslant 1$. Hence $\overleftarrow{\tilde{X}}<_{\text {lex }} \overleftarrow{[i+b-k, i+b-1]}$, which yields $\tilde{X} \in I_{i-1}$.

Case 3.3. $X \in R_{1}$. Then $\bar{X} \leqslant i+b$ and thus $\overline{\tilde{X}} \leqslant i+b-1$. This implies $\overleftarrow{\tilde{X}} \leqslant$ lex $\overleftarrow{[i+b-k, i+b-1]}$ and hence $\tilde{X} \in I_{i-1}$. 
Now the bandwidth of an arbitrary $f_{\text {spo }}$ can be determined:

Lemma 11. We have $B_{f_{s p o}}\left(G_{n, k, b}\right)=\left\lceil\frac{\left|V_{n, k, b}\right|+|C|-2}{2}\right\rceil$.

Proof. We have $f_{\text {spo }}([0, k-1])=1$ and $f_{\text {spo }}([n-k+1, n])=\left|V_{n, k, b}\right|$. Let $u=\left|R_{0}\right|$. Then $f_{\text {spo }}([n-b, n-b+k-1])=u+1$ and $f_{\text {spo }}([b-k+1, b])=u+|C|$. Because of Lemma 9 we have $\left|u-\left(\left|V_{n, k, b}\right|-|C|-u\right)\right| \leqslant 1$ and thus $\left|(u+|C|-1)-\left(\left|V_{n, k, b}\right|-u-1\right)\right| \leqslant 1$. Lemma 10 implies that one of the $f_{\text {spo }}$-distances $d_{f_{s p o}}([0, k-1],[b-k+1, b])=u+|C|-1$ and $d_{f_{s p o}}([n-b, n-b+k-1],[n-k+1, n])=\left|V_{n, k, b}\right|-u-1$ is the maximal $f_{\text {spo }}$-distance. As they both differ from each other by at most 1 it follows that

$$
B_{f_{s p o}}\left(G_{n, k, b}\right)=\left\lceil\frac{u+|C|-1+\left|V_{n, k, b}\right|-u-1}{2}\right\rceil=\left\lceil\frac{\left|V_{n, k, b}\right|+|C|-2}{2}\right\rceil .
$$

Now we are able to prove the part a) of Theorem 1.

Proof of Theorem 1.a). We know from Lemma 11 that $B\left(G_{n, k, b}\right) \leqslant\left[\frac{\left|V_{n, k, b}\right|+|C|-2}{2}\right]$. Let $f$ be an arbitrary proper numbering of $G_{n, k, b}$. Let $X_{V}$ be the vertex with number 1 and $X^{V}$ the vertex with number $\left|V_{n, k, b}\right|$. Further let $X_{C}$ be the vertex of $C$ with smallest number, denoted by $\alpha$, and $X^{C}$ be the vertex of $C$ with largest number, denoted by $\beta$. Then $\beta-\alpha \geqslant|C|-1$. Further $X_{V}$ and $X^{C}$ as well as $X_{C}$ and $X^{V}$ are adjacent with $d_{f}\left(X_{V}, X^{C}\right)=\beta-1$ and $d_{f}\left(X_{C}, X^{V}\right)=\left|V_{n, k, b}\right|-\alpha$. The sum of them is

$$
s=(\beta-1)+\left(\left|V_{n, k, b}\right|-\alpha\right)=\left|V_{n, k, b}\right|+(\beta-\alpha)-1 \geqslant\left|V_{n, k, b}\right|+|C|-2 .
$$

The maximum of both $f$-distances is therefore at least $\left\lceil\frac{\left|V_{n, k, b}\right|+|C|-2}{2}\right\rceil$. From Lemma 8 and (3) we obtain

$$
B\left(G_{n, k, b}\right)=\left\lceil\frac{(n+1)\left(\begin{array}{c}
b \\
k-1
\end{array}\right)-(k-1)\left(\begin{array}{c}
b+1 \\
k
\end{array}\right)+\left(\begin{array}{c}
2 b-n+1 \\
k
\end{array}\right)-2}{2}\right\rceil .
$$

\section{$5 \quad$ Asymptotic bandwidth for $b=o(n)$}

In this section, we consider the case, where $b$ grows sublinearly with respect to $n$. First we take a simple proper numbering, which provides an upper bound for the bandwidth.

Lemma 12. Let $n, k, b$ be arbitrary integers with $1 \leqslant k-1 \leqslant b \leqslant n$. Then

$$
B\left(G_{n, k, b}\right) \leqslant k\left(\begin{array}{l}
b \\
k
\end{array}\right) .
$$

Proof. We order the vertices of $G_{n, k, b}$ in a lexicographic way, see (5). Let $f_{\text {lex }}(X)$ be the label of $X \in V_{n, k, b}$ with respect to this ordering. Now let $X$ and $Y$ be two adjacent vertices with $X<_{\text {lex }} Y$ and let $X^{\prime}=[\underline{X}, \underline{X}+k-1]$ and $Y^{\prime}=[\underline{X}+b-k+1, \underline{X}+b]$. Then

$$
X^{\prime} \leqslant_{\text {lex }} X<_{\text {lex }} Y \leqslant_{\text {lex }} Y^{\prime} .
$$


Moreover, for $j \in[0, n-b]$,

$$
\left|\left\{X \in V_{n, k, b}: \underline{X}=j\right\}\right|=\left(\begin{array}{c}
b \\
k-1
\end{array}\right)
$$

and, for $j \in[n-b+1, n]$,

$$
\left|\left\{X \in V_{n, k, b}: \underline{X}=j\right\}\right| \leqslant\left(\begin{array}{c}
b \\
k-1
\end{array}\right) .
$$

Since $Y^{\prime}$ is the lexicographically smallest vertex with minimum element $\underline{X}+b-k+1$ it follows that

$$
\left|f_{l e x}\left(Y^{\prime}\right)-f_{l e x}\left(X^{\prime}\right)\right| \leqslant(b-k+1)\left(\begin{array}{c}
b \\
k-1
\end{array}\right)=k\left(\begin{array}{l}
b \\
k
\end{array}\right) .
$$

Now (6) and (7) imply

$$
\left|f_{l e x}(Y)-f_{l e x}(X)\right| \leqslant k\left(\begin{array}{l}
b \\
k
\end{array}\right)
$$

which proves the assertion.

Chvátal observed in [6] that a lower bound for the bandwidth is given by

$$
B(G) \geqslant\left\lceil\frac{|V|-1}{\operatorname{diam}(G)}\right\rceil \text {. }
$$

Here the diameter $\operatorname{diam}(G)$ of the graph $G=(V, E)$ is the maximal distance of any two vertices of $G$.

By Lemma 6, the distance of any two vertices of $G_{n, k, b}$ is at most $\left\lceil\frac{n-0-b}{b-k+1}\right\rceil+1=\left\lceil\frac{n-k+1}{b-k+1}\right\rceil$ and by Lemma 5 the vertices $[0, k-1]$ and $[n-k+1, n]$ have distance $\left\lceil\frac{n-k+1}{b-k+1}\right\rceil$. Accordingly,

$$
\operatorname{diam}\left(G_{n, k, b}\right)=\left\lceil\frac{n-k+1}{b-k+1}\right\rceil .
$$

Now we have all preparations to prove Theorem $1 \mathrm{~b}$ ).

Proof of Theorem 1 b). From Lemma 12 we know that

$$
B\left(G_{n, k, b}\right) \leqslant k\left(\begin{array}{l}
b \\
k
\end{array}\right) .
$$

For the lower bound, we use the fact that $\left(\begin{array}{l}b \\ k\end{array}\right)=\frac{b^{k}}{k !}+O\left(b^{k-1}\right)$ as $b \rightarrow \infty$. We have by Lemma 8, (8) and (9) for $n \rightarrow \infty$ :

$$
B\left(G_{n, k, b}\right) \geqslant\left\lceil\frac{\left|V_{n, k, b}\right|-1}{\operatorname{diam}\left(G_{n, k, b}\right)}\right\rceil=\frac{(n-b+1)\left(\begin{array}{c}
b \\
k-1
\end{array}\right)+\left(\begin{array}{l}
b \\
k
\end{array}\right)-1}{\left\lceil\frac{n-k+1}{b-k+1}\right\rceil}=\frac{n\left(\begin{array}{c}
b \\
k-1
\end{array}\right)+O\left(b^{k}\right)}{\frac{n-k+1}{b-k+1}+O(1)}
$$




$$
=\frac{n k\left(\begin{array}{l}
b \\
k
\end{array}\right)+O\left(b^{k+1}\right)}{n+O(b)}=\frac{k\left(\begin{array}{l}
b \\
k
\end{array}\right)+O\left(\frac{b^{k+1}}{n}\right)}{1+O\left(\frac{b}{n}\right)} .
$$

In the case $b=o\left(n^{\frac{1}{k+1}}\right)$ we have

$$
\frac{k\left(\begin{array}{l}
b \\
k
\end{array}\right)+O\left(\frac{b^{k+1}}{n}\right)}{1+O\left(\frac{b}{n}\right)} \geqslant\left(k\left(\begin{array}{l}
b \\
k
\end{array}\right)+o(1)\right)\left(1-O\left(\frac{b}{n}\right)\right)=k\left(\begin{array}{l}
b \\
k
\end{array}\right)-o(1) .
$$

Thus, $B\left(G_{n, k, b}\right) \geqslant k\left(\begin{array}{l}b \\ k\end{array}\right)$ for sufficiently large $n$, which proves the first part of the assertion. If $b=o(n)$, then

$$
\frac{k\left(\begin{array}{l}
b \\
k
\end{array}\right)+O\left(\frac{b^{k+1}}{n}\right)}{1+O\left(\frac{b}{n}\right)} \sim k\left(\begin{array}{l}
b \\
k
\end{array}\right)
$$

which shows that $B\left(G_{n, k, b}\right) \gtrsim k\left(\begin{array}{l}b \\ k\end{array}\right)$ as $n \rightarrow \infty$, which proves the second part of the assertion.

\section{Motivation for the study of polygons}

In the following let $\Omega=\left\{(x, y) \in \mathbb{R}^{2}: 0 \leqslant x \leqslant y \leqslant 1\right\}$. For $X \in V_{n, k, b}$, the point $\frac{1}{n}(\underline{X}, \bar{X})$ belongs to the triangle $\Omega$. Moreover, if $b=\beta n$, then $\frac{1}{n}(\underline{X}, \bar{X}) \in\{(x, y) \in \Omega: y-x \leqslant \beta\}$, i.e., the associated point $\frac{1}{n}(\underline{X}, \bar{X})$ belongs to a trapezoid in $\Omega$.

In Section 9 we prove the lower bounds for the bandwidth by choosing subsets $V^{\prime}$ of $V_{n, k, b}$ in such a way that a set $X$ belongs to $V^{\prime}$ if the associated point $\frac{1}{n}(\underline{X}, \bar{X})$ belongs to a well-defined polygon in $\Omega$. A bound of the bandwidth of the subgraph induced by $V^{\prime}$ yields the lower bound for the bandwidth of $G_{n, k, b}$. In Section 10 we prove the upper bounds for the bandwidth by defining proper numberings of $G_{n, k, b}$ in form of an ordinal sum of well-defined total orders on subsets $V^{\prime}$ of $V$ whose associated points belong to polygons in $\Omega$. These total orders can be interpreted as induced orders of total orders on the whole polygons, but one must take into account that several sets $X \in V_{n, k, b}$ may have the same associated point $\frac{1}{n}(\underline{X}, \bar{X})$.

Thus we need a relationship between the subsets of $V_{n, k, b}$ and the corresponding polygons in $\Omega$. This is established in Section 7. In Section 8 the concrete polygons are introduced and studied.

\section{Asymptotic cardinality of sets defined by polygons}

Let $P$ be a polygon in $\Omega$ and let $\operatorname{int}(P)$ be the interior of $P$. Let

$$
\begin{aligned}
& V_{n, k}(P)=\left\{X \in\left(\begin{array}{c}
{[0, n]} \\
k
\end{array}\right): \frac{1}{n}(\underline{X}, \bar{X}) \in P\right\}, \\
& V_{n, k}^{o}(P)=\left\{X \in\left(\begin{array}{c}
{[0, n]} \\
k
\end{array}\right): \frac{1}{n}(\underline{X}, \bar{X}) \in \operatorname{int}(P)\right\} .
\end{aligned}
$$


Lemma 13. We have

$$
\left|V_{n, k}^{o}(P)\right| \sim\left|V_{n, k}(P)\right| \sim\left(\frac{1}{(k-2) !} \iint_{P}(y-x)^{k-2} d x d y\right) n^{k} \text { as } n \rightarrow \infty .
$$

Proof. Let $i, j$ be integers with $0 \leqslant i \leqslant j \leqslant n$. Obviously,

$$
\left|\left\{X \in\left(\begin{array}{c}
{[0, n]} \\
k
\end{array}\right): \underline{X}=i, \bar{X}=j\right\}\right|=\left(\begin{array}{c}
j-i-1 \\
k-2
\end{array}\right) .
$$

Thus

$$
\left|V_{n, k}(P)\right|=\sum_{\frac{1}{n}(i, j) \in P}\left(\begin{array}{c}
j-i-1 \\
k-2
\end{array}\right)=\sum_{\frac{1}{n}(i, j) \in P}\left(\begin{array}{c}
n(j / n-i / n)-1 \\
k-2
\end{array}\right) .
$$

For $0 \leqslant z \leqslant 1$, we have

$$
\left(\begin{array}{c}
n z-1 \\
k-2
\end{array}\right) \leqslant \frac{z^{k-2}}{(k-2) !} n^{k-2}
$$

Accordingly,

$$
\frac{1}{n^{k}}\left|V_{n, k}(P)\right| \leqslant \frac{1}{(k-2) !} \sum_{\frac{1}{n}(i, j) \in P} \frac{1}{n^{2}}(j / n-i / n)^{k-2} .
$$

The RHS is a Riemann sum for the integral $\iint_{P}(y-x)^{k-2} d x d y$, which shows that

$$
\left|V_{n, k}(P)\right| \lesssim\left(\frac{1}{(k-2) !} \iint_{P}(y-x)^{k-2} d x d y\right) n^{k} \text { as } n \rightarrow \infty .
$$

For $\delta>0$ let $P_{\delta}=\{(x, y) \in P: y-x \geqslant \delta\}$. Clearly,

$$
P_{\delta} \subseteq P .
$$

Obviously, for any $\varepsilon>0$ there is some $\delta>0$ such that

$$
\iint_{P_{\delta}}(y-x)^{k-2} d x d y \geqslant(1-\varepsilon) \iint_{P}(y-x)^{k-2} d x d y .
$$

Moreover, for any $z \geqslant \delta$ there is some $n_{0}$ such that for all $n>n_{0}$

$$
\frac{k-2}{n z} \leqslant \varepsilon \text {. }
$$

This implies

$$
(1-\varepsilon)^{k-2} \frac{(n z)^{k-2}}{(k-2) !} \leqslant\left(1-\frac{k-2}{n z}\right)^{k-2} \frac{(n z)^{k-2}}{(k-2) !} \leqslant\left(\begin{array}{c}
n z-1 \\
k-2
\end{array}\right) .
$$

From (12), (13), (15) and (14) we obtain (with $z=j / n-i / n$ in (15))

$$
\frac{1}{n^{k}}\left|V_{n, k}(P)\right| \geqslant \frac{1}{n^{k}} \sum_{\frac{1}{n}(i, j) \in P_{\delta}}\left(\begin{array}{c}
n(j / n-i / n)-1 \\
k-2
\end{array}\right)
$$




$$
\begin{aligned}
& \geqslant(1-\varepsilon)^{k-2} \frac{1}{(k-2) !} \sum_{\frac{1}{n}(i, j) \in P_{\delta}} \frac{1}{n^{2}}(j / n-i / n)^{k-2} \\
& \gtrsim(1-\varepsilon)^{k-1} \frac{1}{(k-2) !} \iint_{P}(y-x)^{k-2} d x d y .
\end{aligned}
$$

Now, with $\varepsilon \rightarrow 0$ we conclude that

$$
\left|V_{n, k}(P)\right| \gtrsim\left(\frac{1}{(k-2) !} \iint_{P}(y-x)^{k-2} d x d y\right) n^{k} \text { as } n \rightarrow \infty
$$

The reasoning for $\left|V_{n, k}^{o}(P)\right|$ is the same.

For the sake of brevity, we define for fixed $k$ the measure of the polygon $P \subseteq \Omega$ by

$$
\mu(P)=\frac{1}{(k-2) !} \iint_{P}(y-x)^{k-2} d x d y .
$$

Corollary 14. If $S \subseteq\left(\begin{array}{c}{[0, n]} \\ k\end{array}\right)$ is a family of sets that contains all $X \in\left(\begin{array}{c}{[0, n]} \\ k\end{array}\right)$ with $\frac{1}{n}(\underline{X}, \bar{X}) \in \operatorname{int}(P)$ and some $X \in\left(\begin{array}{c}{[0, n]} \\ k\end{array}\right)$ with $\frac{1}{n}(\underline{X}, \bar{X})$ on the boundary of $P$, then

$$
|S| \sim \mu(P) n^{k} \text { as } n \rightarrow \infty .
$$

\section{Definition and measure of crucial polygons}

Recall that we consider the case $b \sim \beta n$ with $\beta \in(0,1]$ and that we use the representation $1=q \beta+r$ with a positive integer $q$ and $0 \leqslant r<\beta$. We define in $\Omega$ several sets of points. First let for $i=1, \ldots, q$

$$
\begin{aligned}
& A_{i}=(i r, i r+q(\beta-r)), \\
& B_{i}=(r-\beta+i \beta, r-\beta+i \beta), \\
& C_{i}=(i \beta, i \beta) .
\end{aligned}
$$

In the following we denote lines given by $y=a x+b$ (or, more generally, by $a x+b y=c$ ) by $g_{y=a x+b}$ (or $g_{a x+b y=c}$ ). Note that the points $B_{i}$ and $C_{i}$ lie on $g_{y=x}$. Furthermore, the points $A_{i}$ lie above or on the line $g_{y=x+\beta}$ iff $r \leqslant \frac{q-1}{q^{2}+q-1}$. This is the reason for the distinction between a) and b) in Theorem 2 .

For Case a), i.e., for $r \leqslant \frac{q-1}{q^{2}+q-1}$, we define points $D_{i}$ and $E_{i}$ as the intersection points of the segments $\overline{A_{i} B_{i}}$ resp. $\overline{A_{i} C_{i}}$ with the line $g_{y=x+\beta}$. An easy computation yields with

$$
\gamma=\beta(1-1 / q)
$$

that for $i=1, \ldots, q$

$$
D_{i}=(r+(i-1) \gamma, r+(i-1) \gamma+\beta)
$$




$$
E_{i}=(i \gamma, i \gamma+\beta)
$$

For Case b), i.e., for $r>\frac{q-1}{q^{2}+q-1}$, we define points $F_{i}$ as those points that provide an equipartition into $q+1$ parts of the segment between $(0,0)$ and $(1,1)$ and points $G_{i}$ as the intersection points of the segments between $F_{i}$ and $(0,1)$ with the line $g_{y=x+\beta}$. It is straightforward that for $i=0, \ldots, q+1$

$$
\begin{aligned}
F_{i} & =(i /(q+1), i /(q+1)), \\
G_{i} & =(i(1-\beta) /(q+1), i(1-\beta) /(q+1)+\beta) .
\end{aligned}
$$

Like in Case a), let $E_{q}$ be the intersection point of the lines $g_{y=x+\beta}$ and $g_{y=q \beta}$. Finally, we define an auxiliary point $H_{1}=(r, \beta)$. For Case a) we also use the points $C_{0}=F_{0}=(0,0)$, $B_{q+1}=F_{q+1}=(1,1), E_{0}=G_{0}=(0, \beta)$ and $D_{q+1}=G_{q+1}=(1-\beta, 1)$.

In Figures 1 and 2 the points are illustrated for $\beta=9 / 20$ (i.e., Case a) with $q=2, r=$ $1 / 10<1 / 5$ ) as well as for $\beta=7 / 20$ (i.e., Case b) with $q=2, r=3 / 10>1 / 5$ ).

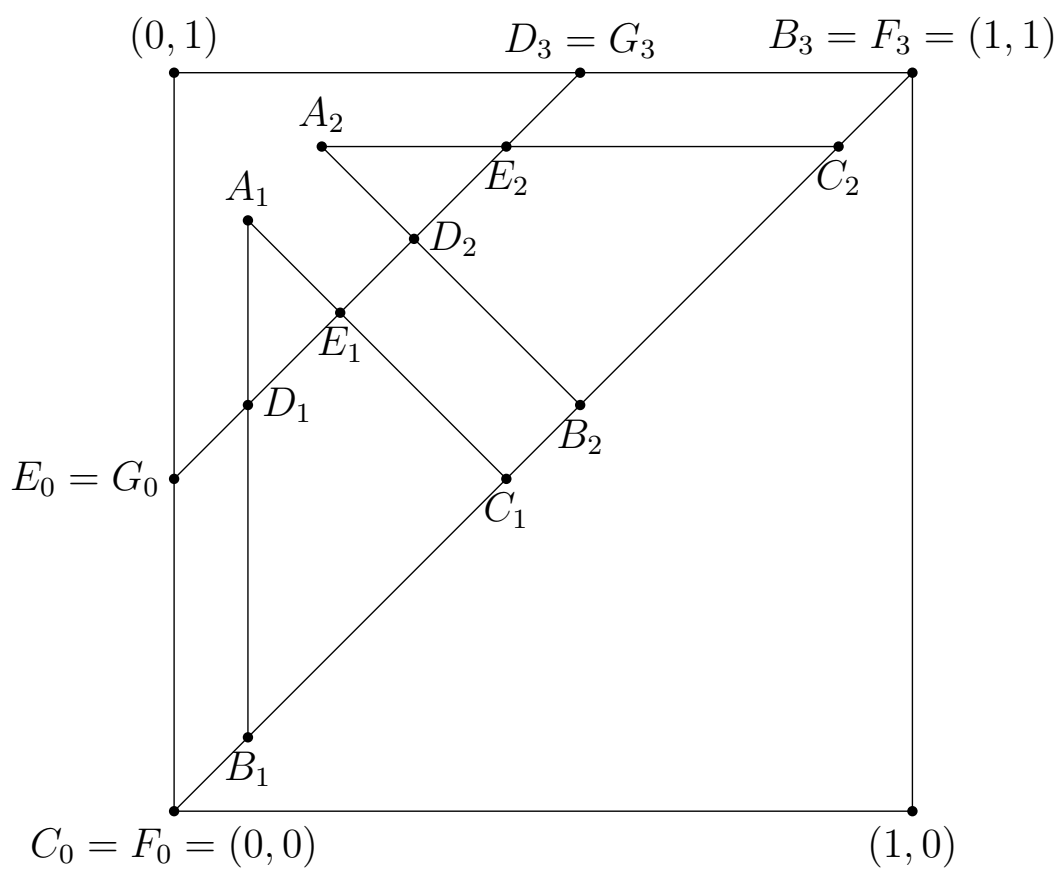

Figure 1: Polygons and important points for $\beta=9 / 20$ (i.e., Case a) with $q=2, r=$ $1 / 10<1 / 5)$.

It is easy to check that the points $A_{i}$ lie on the segments $\overline{F_{i} G_{i}}$ (we have $\overrightarrow{F_{i} A_{i}}=(1-r(q+$ 1) $/ \beta) \overrightarrow{F_{i} G_{i}}$ ) and that the points $F_{0}, B_{1}, F_{1}, C_{1}, \ldots, B_{q}, F_{q}, C_{q}, F_{q+1}$ lie in this order on the line $g_{y=x}$. It is also easy to see that $H_{1}$ lies on the segment $\overline{A_{1} B_{1}}$.

Lemma 15. Let $0 \leqslant s<t \leqslant 1$, let $P_{1}=\left(\xi_{1}, \xi_{1}+s\right), P_{2}=\left(\xi_{2}, \xi_{2}+s\right)$ be points on the line $g_{y=x+s}$ with $0 \leqslant \xi_{1} \leqslant \xi_{2} \leqslant 1-s$ and let $P_{3}=\left(\xi_{3}, \xi_{3}+t\right), P_{4}=\left(\xi_{4}, \xi_{4}+t\right)$ be points on 


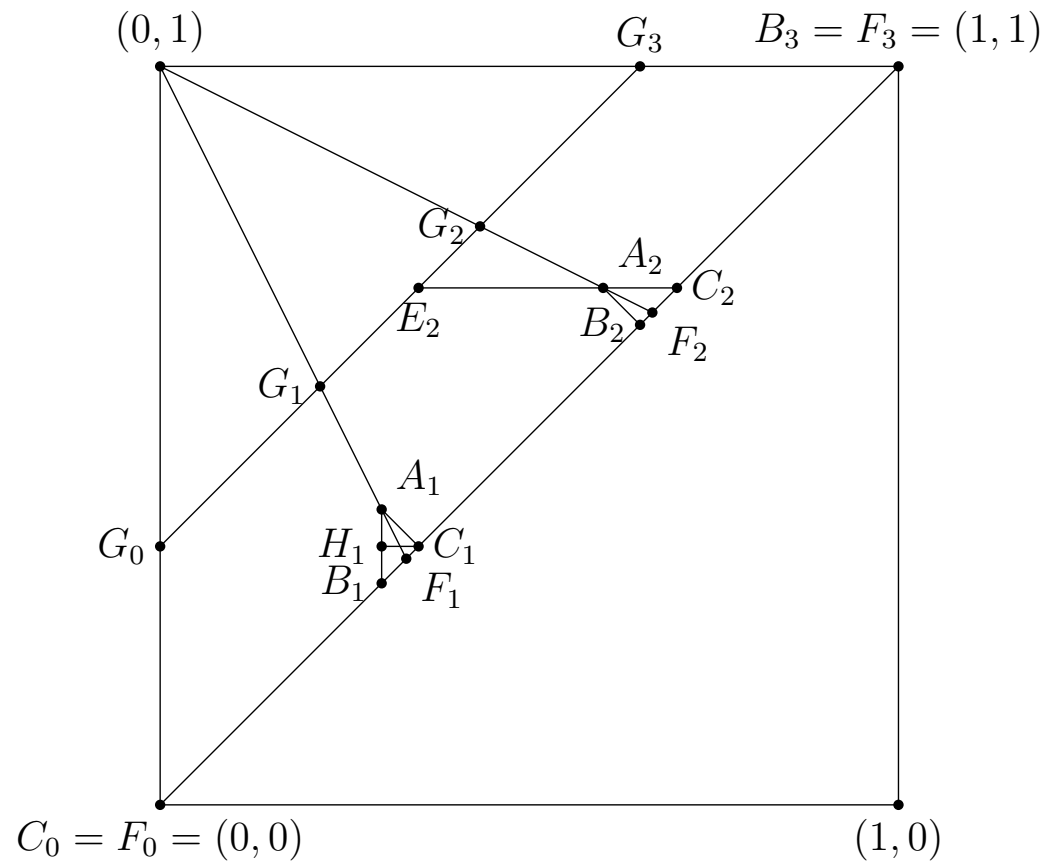

Figure 2: Polygons and important points for $\beta=7 / 20$ (i.e., Case b) with $q=2, r=$ $3 / 10>1 / 5)$.

the line $g_{y=x+t}$ with $0 \leqslant \xi_{3} \leqslant \xi_{4} \leqslant 1-t$. Let $u=\xi_{2}-\xi_{1}$ and $v=\xi_{4}-\xi_{3}$. Then

$$
\mu\left(P_{1} P_{2} P_{4} P_{3}\right)=\frac{1}{(k-2) !} \frac{1}{t-s}\left(\frac{1}{k}(v-u)\left(t^{k}-s^{k}\right)+\frac{t u-s v}{k-1}\left(t^{k-1}-s^{k-1}\right)\right) .
$$

Proof. The proof follows directly by computing the integrals, using the coordinate transformation $x^{\prime}=x+y, y^{\prime}=y-x$. This leads to a domain of integration in form of a trapezoid whose basis is parallel to the $x^{\prime}$-axis.

Since only the difference of the $\xi$-values has influence we have:

Corollary 16. Let $0 \leqslant s<t \leqslant 1$, let $P_{1}, P_{2}$ and $Q_{1}, Q_{2}$ be points of $\Omega$ on the line $g_{y=x+s}$ with $\overrightarrow{P_{1} P_{2}}=\overrightarrow{Q_{1} Q_{2}}$ showing to north east and let $P_{3}, P_{4}$ and $Q_{3}, Q_{4}$ be points on the line $g_{y=x+t}$ with $\overrightarrow{P_{3} P_{4}}=\overrightarrow{Q_{3} Q_{4}}$ showing to north east. Then

$$
\mu\left(P_{1} P_{2} P_{4} P_{3}\right)=\mu\left(Q_{1} Q_{2} Q_{4} Q_{3}\right) .
$$

By inserting the corresponding values and using the definition of the $c$-functions in Theorem 2, we obtain:

Corollary 17. We have for all possible $i$ :

$$
\mu\left(F_{0} F_{q+1} G_{q+1} G_{0}\right)=\frac{\beta^{k-1}}{k !}(k-(k-1) \beta)=(q+1) c_{2}(\beta, k),
$$




$$
\begin{aligned}
\mu\left(C_{i} B_{i+1} D_{i+1} E_{i}\right) & =\frac{\beta^{k-1}}{(k-1) !} r=(q+1) c_{2}(\beta, k)-q c_{1}(\beta, k), \\
\mu\left(B_{i} C_{i} E_{i} D_{i}\right) & =\frac{\beta^{k-1}}{k !}\left(k(\beta-r)-\frac{\beta(k-1)}{q}\right) \\
& =(q+1)\left(c_{1}(\beta, k)-c_{2}(\beta, k)\right), \\
\mu\left(F_{i} F_{i+1} G_{i+1} G_{i}\right) & =\frac{1}{q+1} \mu\left(F_{0} F_{q+1} G_{q+1} G_{0}\right)=c_{2}(\beta, k), \\
\mu\left(A_{i} B_{i} C_{i}\right) & =\frac{(\beta-r)^{k}}{k !} q^{k-1}=(q+1) c_{3}(\beta, k), \\
\mu\left(A_{i} B_{i} F_{i}\right) & =\left(1-\frac{i}{q+1}\right) \mu\left(A_{i} B_{i} C_{i}\right)=(q+1-i) c_{3}(\beta, k), \\
\mu\left(A_{i} F_{i} C_{i}\right) & =\frac{i}{q+1} \mu\left(A_{i} B_{i} C_{i}\right)=i c_{3}(\beta, k), \\
\mu\left(B_{1} C_{1} H_{1}\right) & =\frac{1}{q^{k-1}} \mu\left(A_{1} B_{1} C_{1}\right)=\frac{q+1}{q^{k-1}} c_{3}(\beta, k) .
\end{aligned}
$$

\section{Proof of the lower bounds for the bandwidth in Theorem 2}

Though the following result follows also directly from Lemma 8, we prove it as an example for our polygon-method that will be used in a similar way several times.

Lemma 18. We have

$$
\left|V_{n, k, b}\right| \sim \mu\left(F_{0} F_{q+1} G_{q+1} G_{0}\right) n^{k}=(q+1) c_{2}(\beta, k) n^{k} .
$$

Proof. First let $b=\lfloor\beta n\rfloor$. It is easy to see that $V_{n, k}\left(F_{0} F_{q+1} G_{q+1} G_{0}\right)=V_{n, k, b}$. By Corollaries 14 and 17,

$$
\left|V_{n, k}\left(F_{0} F_{q+1} G_{q+1} G_{0}\right)\right| \sim \mu\left(F_{0} F_{q+1} G_{q+1} G_{0}\right) n^{k}=(q+1) c_{2}(\beta, k) n^{k} .
$$

Now let $\varepsilon>0$ and $\lfloor(\beta-\varepsilon) n\rfloor \leqslant b \leqslant\lfloor(\beta+\varepsilon) n\rfloor$. Then

$$
V_{n, k,\lfloor(\beta-\varepsilon) n\rfloor} \subseteq V_{n, k, b} \subseteq V_{n, k,\lfloor(\beta+\varepsilon) n\rfloor},
$$

hence

$$
(q+1) c_{2}(\beta-\varepsilon, k) n^{k} \lesssim\left|V_{n, k, b}\right| \lesssim(q+1) c_{2}(\beta+\varepsilon, k) n^{k}
$$

and the assertion follows with $\varepsilon \rightarrow 0$ by the continuity of $c_{2}$ as a function of $\beta$.

Now we prove the first asymptotic lower bound. Note that this applies to both Case a) and Case b).

Lemma 19. We have

$$
B\left(G_{n, k, b}\right) \gtrsim \frac{1}{q} \mu\left(F_{0} C_{q} E_{q} G_{0}\right) n^{k}=c_{1}(\beta, k) n^{k} \text { as } n \rightarrow \infty .
$$


Proof. Obviously, $F_{0} C_{q} E_{q} G_{0}=F_{0} F_{q+1} G_{q+1} G_{0} \backslash C_{q} B_{q+1} D_{q+1} E_{q}$ and hence

$$
\mu\left(F_{0} C_{q} E_{q} G_{0}\right)=\mu\left(F_{0} F_{q+1} G_{q+1} G_{0}\right)-\mu\left(C_{q} B_{q+1} D_{q+1} E_{q}\right) .
$$

By Corollary 17,

$$
\mu\left(F_{0} C_{q} E_{q} G_{0}\right)=(q+1) c_{2}(\beta, k)-\left((q+1) c_{2}(\beta, k)-q c_{1}(\beta, k)\right)=q c_{1}(\beta, k)
$$

which shows the last equality in the assertion.

Let $b \sim \beta n$. For $\varepsilon>0$ let $\beta^{\prime}=\beta-\varepsilon, b^{\prime}=\left\lfloor\beta^{\prime} n\right\rfloor$ and $F_{0}^{\prime} C_{q}^{\prime} E_{q}^{\prime} G_{0}^{\prime}$ be the polygon corresponding to $F_{0} C_{q} E_{q} G_{0}$, but depending on $\beta^{\prime}$ instead of $\beta$. We may generally assume that $b \geqslant b^{\prime}$ since this is true if $n$ is sufficiently large. Let briefly $G^{\prime}=G_{n, k, b^{\prime}, b}$, i.e., the vertex set of $G^{\prime}$ is given by

$$
V^{\prime}=\left\{X \in V_{n, k, b}: \frac{1}{n}(\underline{X}, \bar{X}) \in F_{0}^{\prime} C_{q}^{\prime} E_{q}^{\prime} G_{0}^{\prime}\right\} .
$$

Note that $\frac{1}{n} \bar{X} \leqslant q \beta^{\prime}=q \beta(1-\delta)$ with $\delta=\frac{\varepsilon}{\beta}$ for all $X \in V^{\prime}$.

Now we show that for sufficiently large $n$

$$
\operatorname{diam}\left(G^{\prime}\right) \leqslant q .
$$

Let $X$ and $Y$ be any two distinct vertices of $G^{\prime}$. Then $0 \leqslant \underline{X} \leqslant \bar{X} \leqslant q \beta(1-\delta) n$ and $0 \leqslant \underline{Y} \leqslant \bar{Y} \leqslant q \beta(1-\delta) n$. By Lemma $7, X$ and $Y$ have distance at most $q$ if $n$ is sufficiently large, which proves (16). From the Chvátal bound (8) it follows that

$$
B\left(G^{\prime}\right) \geqslant \frac{\left|V^{\prime}\right|-1}{q} \gtrsim \frac{\mu\left(F_{0}^{\prime} C_{q}^{\prime} E_{q}^{\prime} G_{0}^{\prime}\right)}{q} n^{k}=c_{1}(\beta-\varepsilon, k) n^{k} .
$$

By (1), we have $B\left(G_{n, k, b}\right) \geqslant B\left(G^{\prime}\right)$. Since $c_{1}$ is a continuous functions of $\beta$ we obtain with $\varepsilon \rightarrow 0$

$$
B\left(G_{n, k, b}\right) \gtrsim c_{1}(\beta, k) n^{k} .
$$

Now we prove the second asymptotic lower bound, which applies only for Case b).

Lemma 20. If $r>\frac{q-1}{q^{2}+q-1}$, then

$$
\begin{aligned}
B\left(G_{n, k, b}\right) & \gtrsim\left(\mu\left(F_{0} F_{1} G_{1} G_{0}\right)+\frac{1}{q+1} \mu\left(B_{1} C_{1} H_{1}\right)\right) n^{k} \\
& =\left(c_{2}(\beta, k)+\frac{1}{q^{k-1}} c_{3}(\beta, k)\right) n^{k} .
\end{aligned}
$$

Proof. The last equality follows directly from Corollary 17 .

Let $b \sim \beta n$. For $0<\varepsilon<\frac{\beta-r}{q+1}$ let $\beta^{\prime}=\beta-\varepsilon, b^{\prime}=\left\lfloor\beta^{\prime} n\right\rfloor$ and let $F_{0}^{\prime} C_{q}^{\prime} E_{q}^{\prime} G_{0}^{\prime}$ and $R^{\prime}=B_{1}^{\prime} C_{1}^{\prime} H_{1}^{\prime}$ be the polygon corresponding to $F_{0} C_{q} E_{q} G_{0}$ and $R=B_{1} C_{1} H_{1}$, respectively, but depending on $\beta^{\prime}$ instead of $\beta$. The vertices of $R^{\prime}$ are $B_{1}^{\prime}=(r+q \varepsilon, r+q \varepsilon), C_{1}^{\prime}=\left(\beta^{\prime}, \beta^{\prime}\right)$ and $H_{1}^{\prime}=\left(r+q \varepsilon, \beta^{\prime}\right)$. As in the preceding case we may generally assume that $b \geqslant b^{\prime}$. 
Let again $G^{\prime}=G_{n, k, b^{\prime}, b}$ and $V^{\prime}$ be its vertex set. By (1), we have $B\left(G_{n, k, b}\right) \geqslant B\left(G^{\prime}\right)$. Using a distinction of cases we will show that

$$
B\left(G^{\prime}\right) \gtrsim\left(c_{2}(\beta-\varepsilon, k)+\frac{1}{q^{k-1}} c_{3}(\beta-\varepsilon, k)\right) n^{k} .
$$

Since $c_{2}$ and $c_{3}$ are continuous functions of $\beta$ the assertion follows with $\varepsilon \rightarrow 0$.

Let $f$ be a bandwidth numbering of $G^{\prime}$. Let $X_{V^{\prime}}$ and $X^{V^{\prime}}$ be those vertices for which $f\left(X_{V^{\prime}}\right)=1$ and $f\left(X^{V^{\prime}}\right)=\left|V^{\prime}\right|$. Without loss of generality, we may assume that $\underline{X}_{V^{\prime}} \leqslant$ $X^{V^{\prime}}$.

Case 1. $\underline{X}_{V^{\prime}} \leqslant n(r+q \varepsilon)$ and $\underline{X}^{V^{\prime}} \leqslant n(r+q \varepsilon)$ and $q \geqslant 2$.

We have $\varepsilon<\frac{\beta-r}{q+1}$, i.e., $q \varepsilon<\beta^{\prime}-r$ and hence

$$
\max \left\{\bar{X}_{V^{\prime}}, \bar{X}^{V^{\prime}}\right\} \leqslant n\left(r+q \varepsilon+\beta^{\prime}\right) \leqslant 2 \beta^{\prime} n \leqslant 2 \beta(1-\varepsilon) n .
$$

Lemma 7 implies that $X_{V^{\prime}}$ and $X^{V^{\prime}}$ have distance at most 2 and by Lemma 18 and a simple computation, we have

$$
B\left(G^{\prime}\right) \geqslant \frac{\left|V^{\prime}\right|-1}{2} \gtrsim \frac{q+1}{2} c_{2}(\beta-\varepsilon, k) n^{k} \gtrsim\left(c_{2}(\beta-\varepsilon, k)+\frac{1}{q^{k-1}} c_{3}(\beta-\varepsilon, k)\right) n^{k} .
$$

Case 2. $\underline{X}_{V^{\prime}}>n(r+q \varepsilon)$ and $\underline{X}^{V^{\prime}}>n(r+q \varepsilon)$ and $q \geqslant 2$.

Then

$$
\max \left\{\bar{X}^{V^{\prime}}-\underline{X}_{V^{\prime}}, \bar{X}_{V^{\prime}}-\underline{X}^{V^{\prime}}\right\} \leqslant n-n(r+q \varepsilon)=(1-r-q \varepsilon) n=q \beta\left(1-\frac{\varepsilon}{\beta}\right) n .
$$

Lemma 7 implies that $X_{V^{\prime}}$ and $X^{V^{\prime}}$ have distance at most $q$ and by Lemma 18 and a simple computation,

$$
B\left(G^{\prime}\right) \geqslant \frac{\left|V^{\prime}\right|-1}{q} \gtrsim \frac{q+1}{q} c_{2}(\beta-\varepsilon, k) n^{k} \gtrsim\left(c_{2}(\beta-\varepsilon, k)+\frac{1}{q^{k-1}} c_{3}(\beta-\varepsilon, k)\right) n^{k} .
$$

Case 3. $\underline{X}_{V^{\prime}} \leqslant n(r+q \varepsilon)$ and $\underline{X}^{V^{\prime}}>n(r+q \varepsilon)$, or $q=1$.

Let $X_{R^{\prime}}=\arg \min \left\{f(X): X \in V_{n, k}\left(R^{\prime}\right)\right\}$ and $X^{R^{\prime}}=\arg \max \left\{f(X): X \in V_{n, k}\left(R^{\prime}\right)\right\}$. Clearly,

$$
f\left(X^{R^{\prime}}\right)-f\left(X_{R^{\prime}}\right) \geqslant\left|V_{n, k}\left(R^{\prime}\right)\right|-1 .
$$

We will show that

$$
f\left(X^{R^{\prime}}\right)-f\left(X_{V^{\prime}}\right) \leqslant B\left(G^{\prime}\right)
$$

and

$$
f\left(X^{V^{\prime}}\right)-f\left(X_{R^{\prime}}\right) \leqslant q B\left(G^{\prime}\right) .
$$

Then we obtain from (17), (18) and (19)

$$
f\left(X^{V^{\prime}}\right)-f\left(X_{V^{\prime}}\right) \leqslant(q+1) B\left(G^{\prime}\right)-\left(\left|V_{n, k}\left(R^{\prime}\right)\right|-1\right)
$$


and thus by Corollary 17 and Lemma 18,

$$
B\left(G^{\prime}\right) \gtrsim\left(c_{2}(\beta-\varepsilon, k)+\frac{1}{q^{k-1}} c_{3}(\beta-\varepsilon, k)\right) n^{k} .
$$

For the verification of (18) and (19) we distinguish two subcases.

Case 3.1. $q=1$. Let $X \in V_{n, k}\left(R^{\prime}\right)$ and $Y \in V^{\prime}$. It is sufficient to show that $X$ and $Y$ are adjacent using Lemma 4. Indeed, we have $n(r+\varepsilon) \leqslant \underline{X}$ and $\bar{X} \leqslant n(\beta-\varepsilon)$ and consequently, $\bar{Y}-\underline{X} \leqslant n(1-r-\varepsilon)=n \beta^{\prime}$, i.e., $\bar{Y}-\underline{X} \leqslant b^{\prime} \leqslant b$, as well as $\bar{X}-\underline{Y} \leqslant n(\beta-\varepsilon-0)=n \beta^{\prime}$, i.e., $\bar{X}-\underline{Y} \leqslant b^{\prime} \leqslant b$.

Case 3.2. $q \geqslant 2$. Then $\underline{X}_{V^{\prime}} \leqslant n(r+q \varepsilon)$ and $\underline{X}^{V^{\prime}}>n(r+q \varepsilon)$. In view of $n(r+$ $q \varepsilon) \leqslant \bar{X}_{R^{\prime}} \leqslant n \beta^{\prime}, \underline{X}_{V^{\prime}} \geqslant 0, \bar{X}_{V^{\prime}} \leqslant n(r+q \varepsilon)+b^{\prime}$ we have $\bar{X}_{R^{\prime}}-\underline{X}_{V^{\prime}} \leqslant b^{\prime} \leqslant b$ and $\bar{X}_{V^{\prime}}-\underline{X}_{R^{\prime}} \leqslant b^{\prime} \leqslant b$. Thus, by Lemma $4, X_{V^{\prime}}$ and $X_{R^{\prime}}$ are adjacent which implies (18). In view of $\underline{X}_{R^{\prime}}, \underline{X}^{V^{\prime}} \geqslant n(r+q \varepsilon)$ and analogously to the beginning of Case 2, $X_{R^{\prime}}$ and $X^{V^{\prime}}$ have distance at most $q$ which implies (19).

\section{Proof of the upper bounds for the bandwidth in Theorem 2}

In the following, we present two proper numberings $f$ of $G_{n, k, b}$ whose bandwidth is asymptotically equal to the asserted upper bounds. First we only consider the case $b=\lfloor\beta n\rfloor$ since the vertex set $V_{n, k, b}$ is projected optimally into the polygon $F_{0} F_{q+1} G_{q+1} G_{0}$ in this case.

Later we show the general case $b \sim \beta n$ by using this special case and limits. As in Section 4, we define a total order $V_{n, k, b}=S_{1} \oplus \cdots \oplus S_{l}$ with suborders given by means of polygons. In order to avoid intersections on the boundaries we explicitly describe which part of the boundary is deleted, though the ordering can be given on the whole polygon. For example, a notation of the form $C_{0} B_{1} D_{1} E_{0} \backslash \overline{B_{1} D_{1}}$ means that the segment $\overline{B_{1} D_{1}}$ is deleted from the closed quadrangle $C_{0} B_{1} D_{1} E_{0}$.

Case a) $r \leqslant \frac{q-1}{q^{2}+q+1}$.

Using the definition of $V_{n, k}(P)$ in $(10)$ we define the total order $\leqslant$ as follows:

$$
\begin{aligned}
& V_{n, k, b}=V_{n, k}\left(C_{0} B_{1} D_{1} E_{0} \backslash \overline{B_{1} D_{1}}\right) \oplus V_{n, k}\left(B_{1} C_{1} E_{1} D_{1} \backslash \overline{C_{1} E_{1}}\right) \\
& \oplus V_{n, k}\left(C_{1} B_{2} D_{2} E_{1} \backslash \overline{B_{2} D_{2}}\right) \oplus \ldots \\
& \oplus V_{n, k}\left(B_{q} C_{q} E_{q} D_{q} \backslash \overline{C_{q} E_{q}}\right) \oplus V_{n, k}\left(C_{q} B_{q+1} D_{q+1} E_{q}\right) .
\end{aligned}
$$

We still have to define the ordering of the elements of $V_{n, k}\left(C_{i} B_{i+1} D_{i+1} E_{i}\right), i=0, \ldots, q$, and of $V_{n, k}\left(B_{i} C_{i} E_{i} D_{i}\right), i=1, \ldots, q$ (here we may allow the complete boundary).

If $(\underline{X}, \bar{X})=(\underline{Y}, \bar{Y})$, we set in both cases $X \leqslant_{i} Y$ if $X \leqslant_{l e x} Y$. Thus let $(\underline{X}, \bar{X}) \neq(\underline{Y}, \bar{Y})$. First we discuss $V_{n, k}\left(C_{i} B_{i+1} D_{i+1} E_{i}\right)$. We use a new coordinate system with the same origin and with transformation matrix and inverse transformation matrix

$$
M_{i}=\left(\begin{array}{cc}
1 & -i / q \\
1 & 1-i / q
\end{array}\right) \text { and } M_{i}^{-1}=\left(\begin{array}{cc}
1-i / q & i / q \\
-1 & 1
\end{array}\right)
$$


Thus the new coordinate axes have direction of $\overrightarrow{C_{i} B_{i+1}}$ and $\overrightarrow{C_{i} E_{i}}$. The ordering is a lexicographic ordering of the points $\frac{1}{n}(\underline{X}, \bar{X})$ with respect to the new coordinate system, i.e., for $X, Y \in V_{n, k}\left(C_{i} B_{i+1} D_{i+1} E_{i}\right)$ and $(\underline{X}, \bar{X}) \neq(\underline{Y}, \bar{Y})$ we set

$$
X \leqslant_{i} Y \text { if }((1-i / q) \underline{X}+i \bar{X} / q,-\underline{X}+\bar{X}) \leqslant_{l e x}((1-i / q) \underline{Y}+i \bar{Y} / q,-\underline{Y}+\bar{Y}),
$$

see Figure 3.

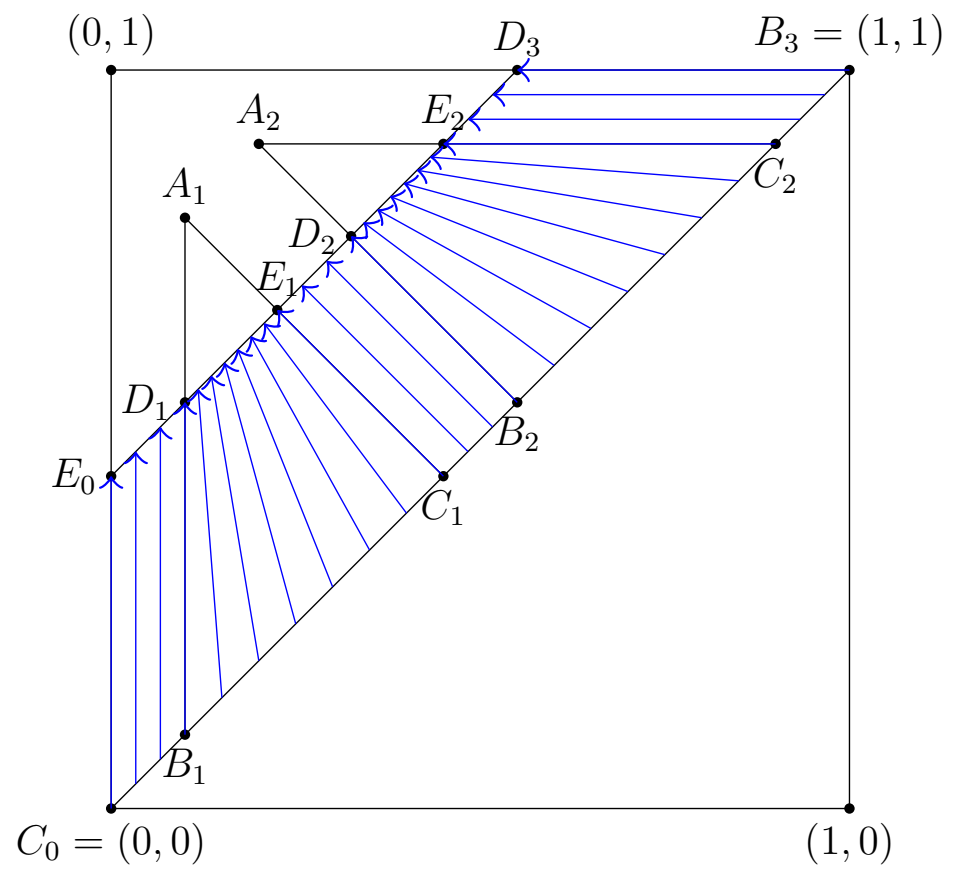

Figure 3: Schematic illustration of the ordering for Case a).

Now we discuss $V_{n, k}\left(B_{i} C_{i} E_{i} D_{i}\right)$. Here we work with polar coordinates in the coordinate system with origin $A_{i}$ and $x$-axis in the direction of $\overrightarrow{A_{i} B_{i}}$ and arbitrary, but fixed unit length. For $X \in V_{n, k}\left(B_{i} C_{i} E_{i} D_{i}\right)$ let $\varphi_{i}(X)$ and $r_{i}(X)$ be the angular and radial coordinates of $\frac{1}{n}(\underline{X}, \bar{X})$ in this coordinate system. The ordering is a lexicographic ordering with respect to the reflected polar coordinates, i.e., for $X, Y \in V_{n, k}\left(B_{i} C_{i} E_{i} D_{i}\right)$ and $(\underline{X}, \bar{X}) \neq(\underline{Y}, \bar{Y})$ we set

$$
X \leqslant_{i} Y \text { if }\left(\varphi_{i}(X),-r_{i}(X)\right) \leqslant_{l e x}\left(\varphi_{i}(Y),-r_{i}(Y)\right)
$$

see Figure 3.

Note that for simpler numerical computations $\varphi_{i}(X)$ may be enlarged to an angle such that one leg is parallel to the $y$-axis, the size of the angle may be replaced by $\tan \left(\varphi_{i}(X)\right)$ and the Euclidean norm for $r_{i}(X)$ may be replaced by some other norm, e.g. the $L_{1}$-norm. It is easy to check that

$$
X \leqslant Y \text { implies } \underline{X} \leqslant \bar{Y} \text {. }
$$

Lemma 21. Let $f_{a}$ be the numbering for Case a). Then, for $b=\lfloor\beta n\rfloor$ and $n \rightarrow \infty$,

$$
B_{f_{a}}(G) \lesssim c_{1}(\beta, k) n^{k}
$$


Proof. Let $P=(\xi, \xi)$ be any point on the segment $\overline{C_{0} B_{q+1}}$, i.e., $0 \leqslant \xi \leqslant 1$. With $P$ we associate a new point $\hat{P}$ as follows: If $P \in \overline{C_{i} B_{i+1}}$ for some $i$, then let $\hat{P}$ be the intersection point of the line $g_{y=x+\beta}$ with the line through $P$ that is parallel to $C_{i} E_{i}$. If $P \in \overline{B_{i} C_{i}}$ for some $i$, then let $\hat{P}$ be the intersection point of the line $g_{y=x+\beta}$ with the line through $P$ and $A_{i}$, see Figure 4 .

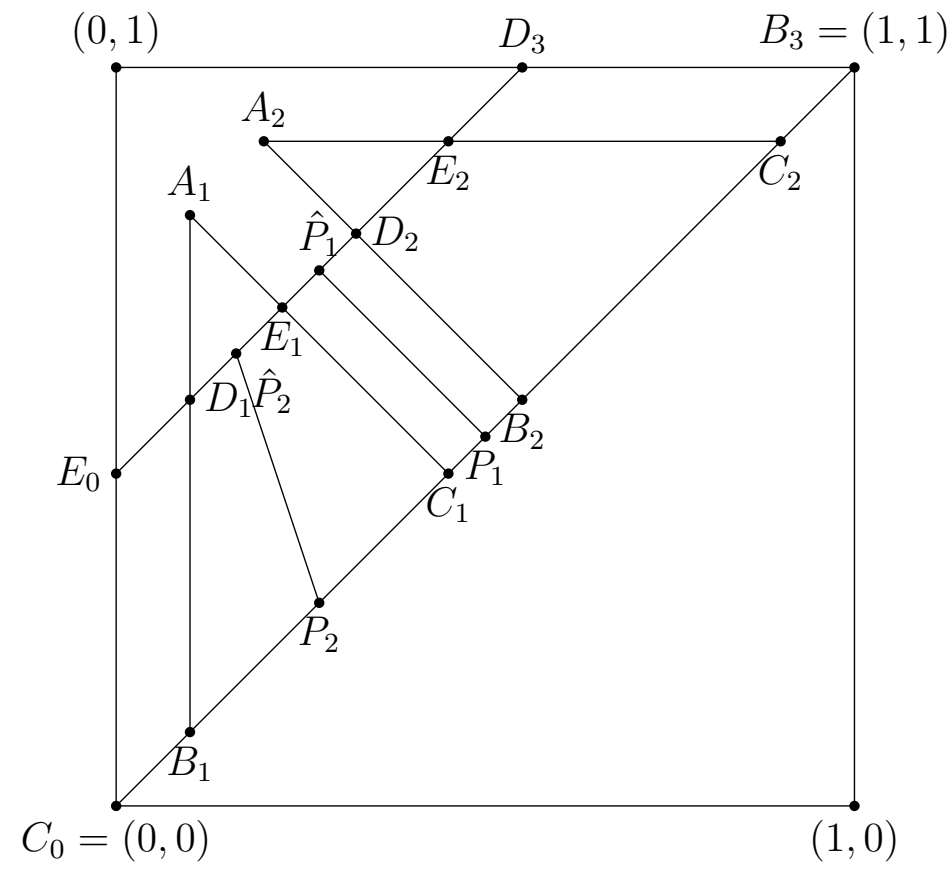

Figure 4: Important points for the upper bound for Case a).

Moreover, for $X \in V_{n, k, b}$ let

$$
P_{\underline{X}}=\frac{1}{n}(\underline{X}, \underline{X}) \text { and } P_{\bar{X}}=\frac{1}{n}(\bar{X}, \bar{X}) .
$$

Let $X, Y \in V_{n, k, b}$ with $X \leqslant Y$. By the definition of the ordering and in view of (20),

$$
f_{a}(Y)-f_{a}(X) \leqslant\left|V_{n, k}\left(P_{\underline{X}} P_{\bar{Y}} \hat{P}_{\bar{Y}} \hat{P}_{\underline{X}}\right)\right| .
$$

Thus we have to prove that

$$
\left|V_{n, k}\left(P_{\underline{X}} P_{\bar{Y}} \hat{P}_{\bar{Y}} \hat{P}_{\underline{X}}\right)\right| \lesssim c_{1}(\beta, k) n^{k} .
$$

Note that

$$
\overrightarrow{B_{i} B_{i+1}}=\overrightarrow{C_{i} C_{i+1}}=(\beta, \beta) .
$$

Since $X$ and $Y$ are adjacent we have by Lemma $4, \bar{Y}-\underline{X} \leqslant b \leqslant \beta n$. Let

$$
P^{\prime}=\left(\frac{1}{n} \underline{X}+\beta, \frac{1}{n} \underline{X}+\beta\right) .
$$




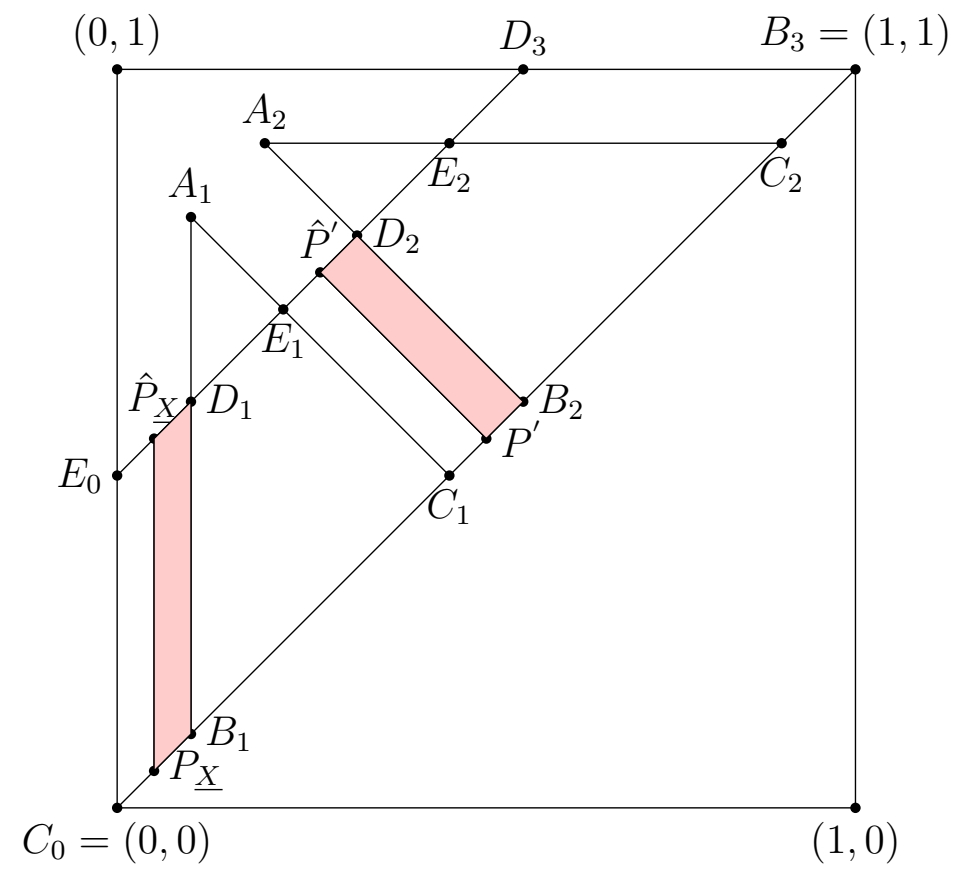

Figure 5: Illustration of (25), where $P_{\underline{X}} \in \overline{C_{i} B_{i+1}}$ for some $i$.

Then

$$
\mu\left(P_{\underline{X}} P_{\bar{Y}} \hat{P}_{\bar{Y}} \hat{P}_{\underline{X}}\right) \leqslant \mu\left(P_{\underline{X}} P^{\prime} \hat{P}^{\prime} \hat{P}_{\underline{X}}\right) .
$$

By Corollaries 16 and 17 we get for all possible $i$,

$$
\begin{aligned}
\mu\left(P_{\underline{X}} P^{\prime} \hat{P}^{\prime} \hat{P}_{\underline{X}}\right) & =\mu\left(B_{i} B_{i+1} D_{i+1} D_{i}\right)=\mu\left(C_{i} C_{i+1} E_{i+1} E_{i}\right) \\
& =\mu\left(B_{i} C_{i} E_{i} D_{i}\right)+\mu\left(C_{i} B_{i+1} D_{i+1} E_{i}\right) \\
& =c_{1}(\beta, k) .
\end{aligned}
$$

An illustration of this fact can be found in Figures 5 and 6 . Both red quadrangles have the same measure by Corollary 16 and the intercept theorem.

By Corollary 14,

$$
\left|V_{n, k}\left(P_{\underline{X}} P_{\bar{Y}} \hat{P}_{\bar{Y}} \hat{P}_{\underline{X}}\right)\right| \lesssim\left(c_{1}(\beta, k)\right) n^{k},
$$

and we get $(22)$.

Case b) $r>\frac{q-1}{q^{2}+q+1}$.

For this case, we use an ordering similar to Case a), but with different polygons, due to the different location of their defining points. Let $\left.A_{0}=(0, q(\beta-r)), A_{q+1}=((q+1) r, 1)\right)$ and $I=(0,1)$. Note that the points $A_{i}, i=0, \ldots, q+1$, lie on the line $g_{y=x+q(\beta-r)}$. We define the total order $\leqslant$ as follows:

$$
\begin{aligned}
V_{n, k, b}= & V_{n, k}\left(C_{0} B_{1} A_{1} G_{1} G_{0} A_{0} \backslash\left(\overline{A_{1} B_{1}} \cup \overline{A_{1} G_{1}}\right)\right) \oplus V_{n, k}\left(A_{1} B_{1} C_{1} \backslash \overline{A_{1} C_{1}}\right) \\
& \oplus V_{n, k}\left(C_{1} B_{2} A_{2} G_{2} G_{1} A_{1} \backslash\left(\overline{A_{2} B_{2}} \cup \overline{A_{2} G_{2}}\right)\right) \oplus \cdots
\end{aligned}
$$




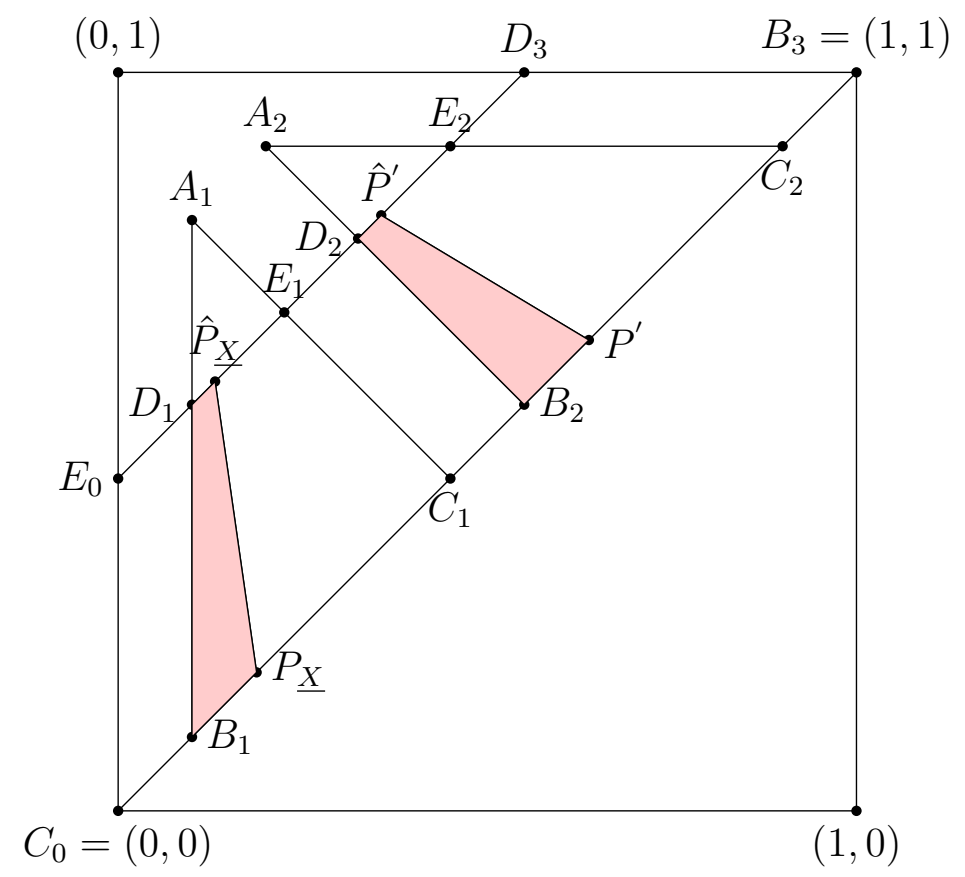

Figure 6: Illustration of (25), where $P_{\underline{X}} \in \overline{B_{i} C_{i}}$ for some $i$.

$$
\begin{aligned}
& \oplus V_{n, k}\left(C_{q-1} B_{q} A_{q} G_{q} G_{q-1} A_{q-1} \backslash\left(\overline{A_{q} B_{q}} \cup \overline{A_{q} G_{q}}\right)\right) \\
& \oplus V_{n, k}\left(A_{q} B_{q} C_{q} \backslash \overline{A_{q} C_{q}}\right) \oplus V_{n, k}\left(C_{q} B_{q+1} G_{q+1} G_{q} A_{q}\right) .
\end{aligned}
$$

We still have to define the ordering of the elements of $V_{n, k}\left(C_{i} B_{i+1} A_{i+1} G_{i+1} G_{i} A_{i}\right), i=$ $0, \ldots, q$, and of $V_{n, k}\left(A_{i} B_{i} C_{i}\right), i=1, \ldots, q$ (again, we may allow the complete boundary). If $(\underline{X}, \bar{X})=(\underline{Y}, \bar{Y})$, we set in all cases $X \leqslant_{i} Y$ if $X \leqslant_{\text {lex }} Y$. Thus let $(\underline{X}, \bar{X}) \neq(\underline{Y}, \bar{Y})$.

First we discuss $V_{n, k}\left(C_{i} B_{i+1} A_{i+1} G_{i+1} G_{i} A_{i}\right)$. We divide the hexagon $C_{i} B_{i+1} A_{i+1} G_{i+1} G_{i} A_{i}$ into two quadrangles $C_{i} B_{i+1} A_{i+1} A_{i}$ and $A_{i} A_{i+1} G_{i+1} G_{i}$, define the corresponding orderings for both quadrangles and then explain how they are combined.

The definition of the ordering of $V_{n, k}\left(C_{i} B_{i+1} A_{i+1} A_{i}\right)$ is similar to $V_{n, k}\left(C_{i} B_{i+1} D_{i+1} E_{i}\right)$ of Case a), i.e., for $X, Y \in V_{n, k}\left(C_{i} B_{i+1} A_{i+1} A_{i}\right)$ and $(\underline{X}, \bar{X}) \neq(\underline{Y}, \bar{Y})$ we set

$$
X \leqslant_{i} Y \text { if }((1-i / q) \underline{X}+i \bar{X} / q,-\underline{X}+\bar{X}) \leqslant_{l e x}((1-i / q) \underline{Y}+i \bar{Y} / q,-\underline{Y}+\bar{Y}) .
$$

Concerning $V_{n, k}\left(A_{i} A_{i+1} G_{i+1} G_{i}\right)$ we work for $X \in V_{n, k}\left(A_{i} A_{i+1} G_{i+1} G_{i}\right)$ with polar coordinates $\varphi_{i}(X)$ and $r_{i}(X)$ of points $\frac{1}{n}(\underline{X}, \bar{X})$ in the coordinate system with origin $I$ and $x$-axis in the direction of $\overrightarrow{I A_{i}}$ and arbitrary, but fixed unit length. Similarly to $V_{n, k}\left(B_{i} C_{i} E_{i} D_{i}\right)$ in Case a), the ordering is a lexicographic ordering with respect to the reflected polar coordinates, i.e., for $X, Y \in V_{n, k}\left(A_{i} A_{i+1} G_{i+1} G_{i}\right)$ and $(\underline{X}, \bar{X}) \neq(\underline{Y}, \bar{Y})$ we set

$$
X \leqslant_{i} Y \text { if }\left(\varphi_{i}(X),-r_{i}(X)\right) \leqslant_{l e x}\left(\varphi_{i}(Y),-r_{i}(Y)\right) .
$$

For a point $P \in C_{i} B_{i+1} A_{i+1} A_{i}$ let $\tilde{P}$ be the intersection point of the line $g_{y=x+q(\beta-r)}$ with the line through $P$ that is parallel to $C_{i} A_{i}$. If, in particular, $P=\frac{1}{n}(\underline{X}, \bar{X})$ with 
$X \in V_{n, k}\left(C_{i} B_{i+1} A_{i+1} A_{i}\right)$, then let $\tilde{\varphi}(X)$ be the angular coordinate of $\tilde{P}$ in the coordinate system introduced for $V_{n, k}\left(A_{i} A_{i+1} G_{i+1} G_{i}\right)$.

The combination of the two orderings is as follows: Let $X \in V_{n, k}\left(C_{i} B_{i+1} A_{i+1} A_{i}\right)$ and $Y \in V_{n, k}\left(A_{i} A_{i+1} G_{i+1} G_{i}\right)$ and $(\underline{X}, \bar{X}) \neq(\underline{Y}, \bar{Y})$. We set

$$
X \leqslant_{i} Y \text { if } \tilde{\varphi}_{i}(X) \leqslant \varphi_{i}(Y) .
$$

Finally, we discuss $V_{n, k}\left(A_{i} B_{i} C_{i}\right)$. Here the ordering is a lexicographic ordering of the reflected polar coordinates in the coordinate system with origin $A_{i}$ and $x$-axis in the direction of $\overrightarrow{A_{i} B_{i}}$ and arbitrary, but fixed unit length.

The whole ordering is illustrated in Figure 7.

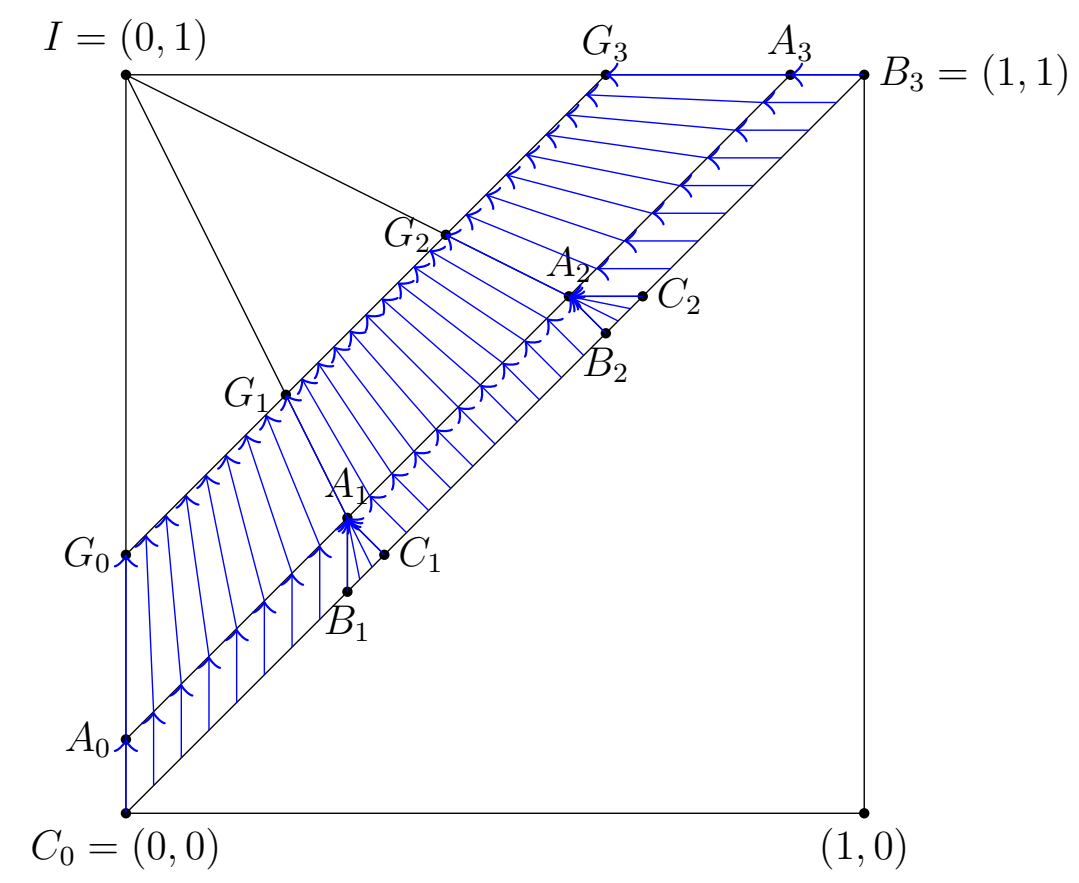

Figure 7: Schematic illustration of the ordering for Case b).

Lemma 22. Let $f_{b}$ be the numbering for Case b). Then

$$
B_{f_{b}}\left(G_{n, k, b}\right) \lesssim\left(c_{2}(\beta, k)+c_{3}(\beta, k)\right) n^{k},
$$

with $b=\lfloor\beta n\rfloor$ as $n \rightarrow \infty$.

Proof. Let $P=(\xi, \xi)$ be any point on the segment $\overline{C_{0} B_{q+1}}$, i.e., $0 \leqslant \xi \leqslant 1$. With $P$ we associate a new point $\hat{P}$ as follows: If $P \in \overline{C_{i} B_{i+1}}$ for some $i$, i.e., $P$ belongs to the quadrangle $C_{i} B_{i+1} A_{i+1} A_{i}$, then we already defined $\tilde{P}$. The point $\hat{P}$ is the intersection point of the line $g_{y=x+\beta}$ with the line through $\tilde{P}$ and $I$. If $P \in \overline{B_{i} C_{i}}$ for some $i$, then let $\hat{P}$ be the intersection point of the line $g_{y=x+\beta}$ with the line through $I$ and $A_{i}$, see Figure 8 . 


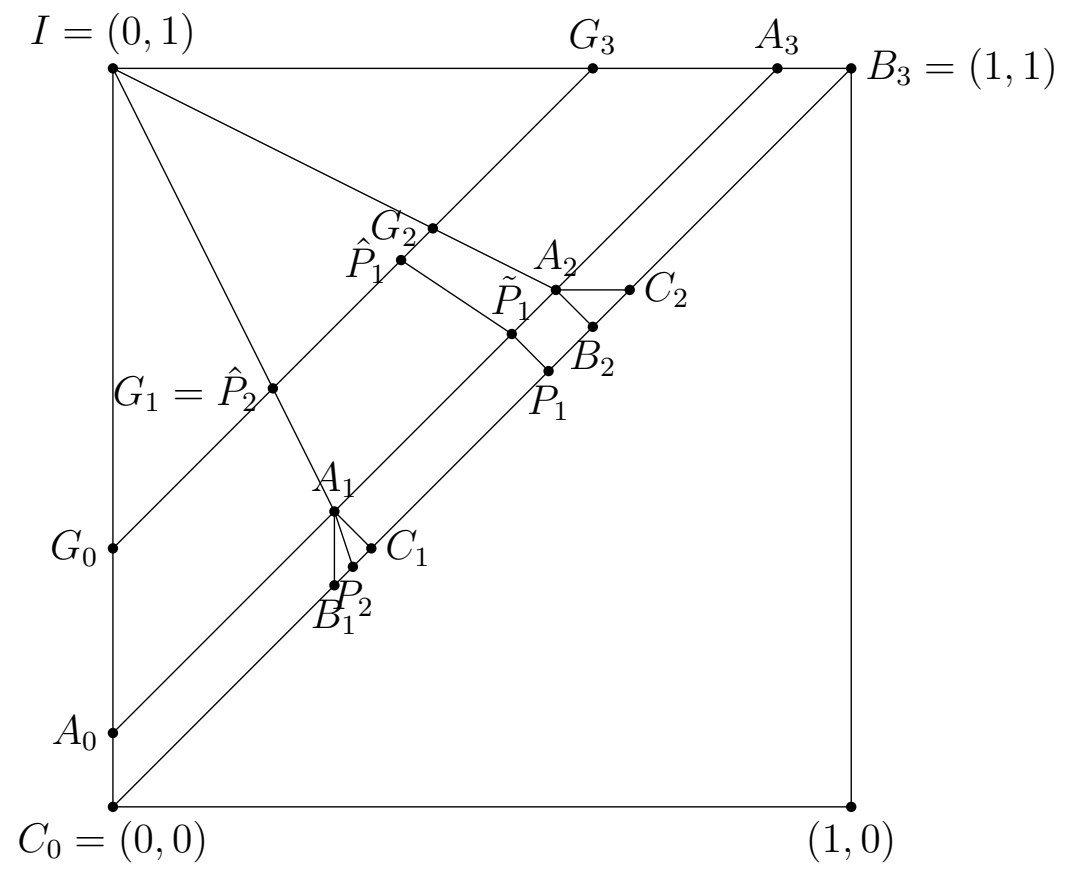

Figure 8: Important points for the upper bound for Case b).

For $X \in V_{n, k, b}$ we define $P_{\underline{X}}$ and $P_{\bar{X}}$ as in (21) and, analogously to Case a), have to prove that for $n \rightarrow \infty$

$$
\left|V_{n, k}\left(P_{\underline{X}} P_{\bar{Y}} \hat{P}_{\bar{Y}} \hat{P}_{\underline{X}}\right)\right| \lesssim\left(c_{2}(\beta, k)+c_{3}(\beta, k)\right) n^{k} .
$$

We define $P^{\prime}$ as in (24). With the same arguments as for Case a) it is sufficient to prove that

$$
\mu\left(P_{\underline{X}} P^{\prime} \hat{P}^{\prime} \hat{P}_{\underline{X}}\right)=c_{2}(\beta, k)+c_{3}(\beta, k) .
$$

Using Lemma 17, one can verify that for all possible $i$,

$$
\begin{aligned}
\mu\left(P_{\underline{X}} P^{\prime} \hat{P}^{\prime} \hat{P}_{\underline{X}}\right) & =\mu\left(B_{i} B_{i+1} A_{i+1} G_{i+1} G_{i} A_{i}\right)=\mu\left(C_{i} C_{i+1} A_{i+1} G_{i+1} G_{i} A_{i}\right) \\
& =\mu\left(F_{i} F_{i+1} G_{i+1} G_{i}\right)+\mu\left(A_{i+1} F_{i+1} C_{i+1}\right)-\mu\left(A_{i} F_{i} C_{i}\right) \\
& =c_{2}(\beta, k)+c_{3}(\beta, k) .
\end{aligned}
$$

An illustration of this fact can be found in Figures 9 and 10.

Now we prove the upper bounds in the general case $b \sim \beta n$. As a tool, we need the following relation between $c_{2}(\beta, k)+c_{3}(\beta, k)$ and $c_{1}(\beta, k)$ on the set with the remainder $r=0$, i.e., on

$$
M:=\{\beta \in(0,1): \exists q \in \mathbb{N} \backslash\{0,1\} \text { such that } 1=q \beta\} .
$$

Lemma 23. We have for all $\beta \in M$

$$
\lim _{\beta^{\prime} \backslash \beta}\left(c_{2}\left(\beta^{\prime}, k\right)+c_{3}\left(\beta^{\prime}, k\right)\right)=c_{1}(\beta, k) .
$$




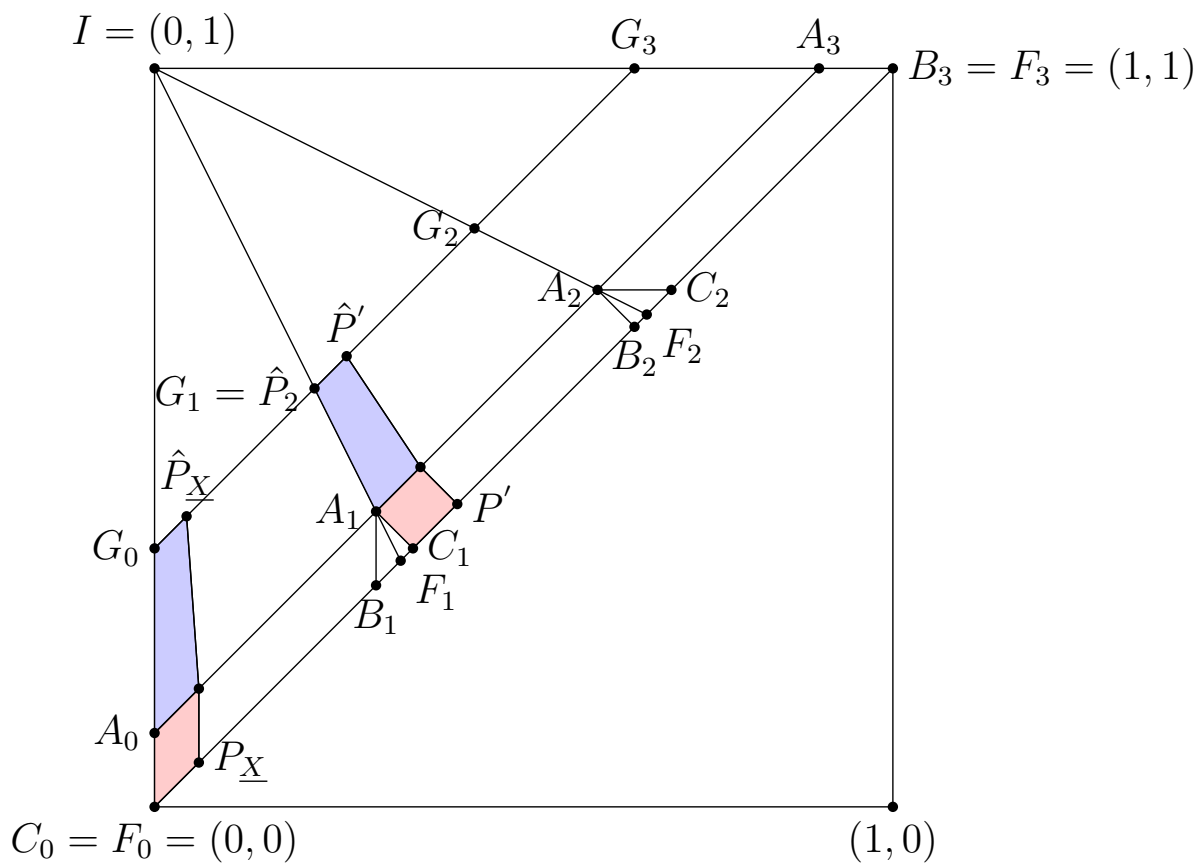

Figure 9: Illustration of (27), where $P_{\underline{X}} \in \overline{C_{i} B_{i+1}}$ for some $i$. Both red and both blue quadrangles have the same measure by Corollary 16 and the intercept theorem.

Proof. Let $\beta \in M$. Then $1=q \beta$ for some $q \geqslant 2$. Further let $\beta^{\prime}>\beta$ with $1=(q-1) \beta^{\prime}+r^{\prime}$ and $r^{\prime}>\frac{(q-1)-1}{(q-1)^{2}+(q-1)-1}$. We consider $r^{\prime}=1-(q-1) \beta^{\prime}$ as a function of $\beta^{\prime}$. Then

$$
\lim _{\beta^{\prime} \searrow \beta} r^{\prime}=\beta
$$

and hence

$$
\lim _{\beta^{\prime} \searrow \beta} c_{3}\left(\beta^{\prime}, k\right)=\lim _{\beta^{\prime} \searrow \beta} \frac{\left(\beta^{\prime}-r^{\prime}\right)^{k}}{((q-1)+1) k !}(q-1)^{k-1}=0 .
$$

Moreover

$$
\begin{aligned}
\lim _{\beta^{\prime} \backslash \beta} c_{2}\left(\beta^{\prime}, k\right) & =\lim _{\beta^{\prime} \searrow \beta} \frac{\left(\beta^{\prime}\right)^{k-1}}{((q-1)+1) k !}\left(k-(k-1) \beta^{\prime}\right)=\lim _{\beta^{\prime} \searrow \beta} \frac{\left(\beta^{\prime}\right)^{k}}{q k !}\left(\frac{k}{\beta^{\prime}}-(k-1)\right) \\
& =\frac{\beta^{k}}{q k !}\left(\frac{k}{\beta}-(k-1)\right)=\frac{\beta^{k}}{k !}\left(k-\frac{k-1}{q}\right)=c_{1}(\beta, k) .
\end{aligned}
$$

The sum of these limits yields the assertion.

The next corollary settles Case a).

Corollary 24. Let $k \geqslant 2$ be an integer, $b \sim \beta n$ for $n \rightarrow \infty$ and $1=q \beta+r$ with a positive integer $q$ and let $0 \leqslant r \leqslant \frac{q-1}{q^{2}+q-1}$. Then

$$
B\left(G_{n, k, b}\right) \lesssim c_{1}(\beta, k) n^{k}
$$




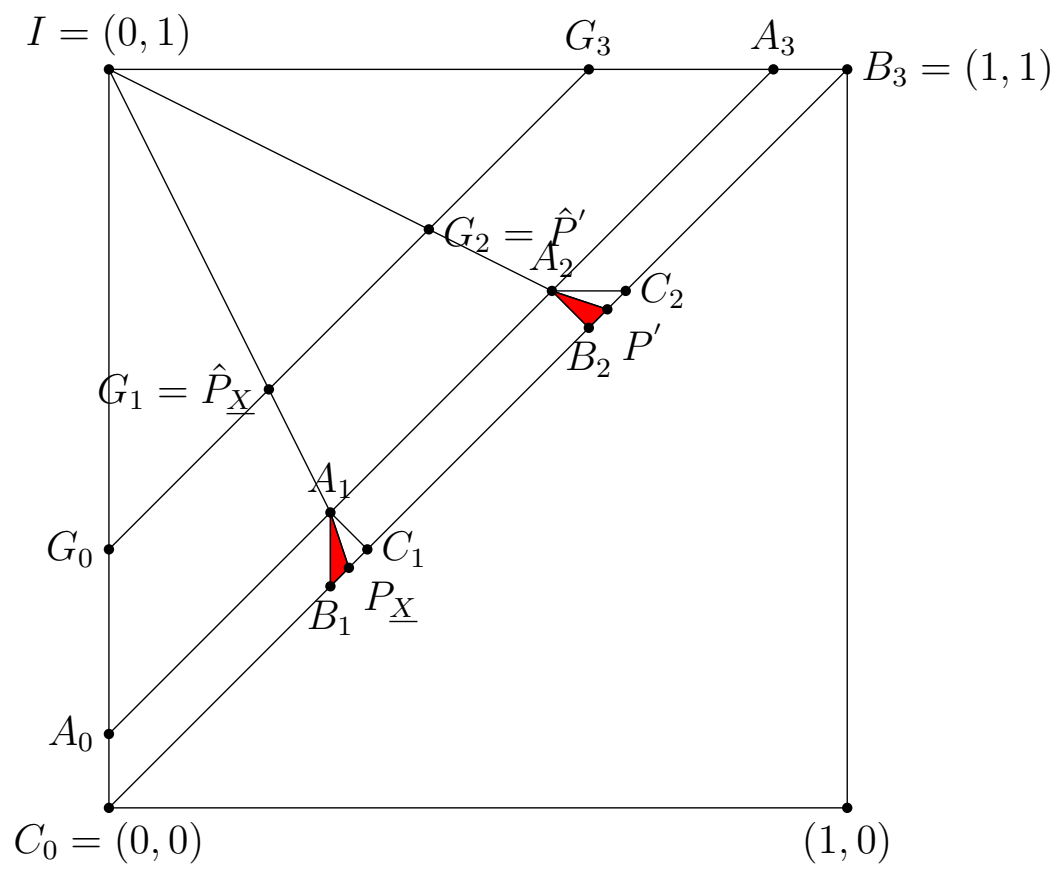

Figure 10: Illustration of (27), where $P_{\underline{X}} \in \overline{B_{i} C_{i}}$ for some $i$. Both red quadrangles have the same measure by Corollary 16.

Proof. First let $r>0$. For $0<\varepsilon<\frac{r}{q}$ let $\beta^{\prime}=\beta+\varepsilon, b^{\prime}=\left\lfloor\beta^{\prime} n\right\rfloor$. Then $r^{\prime}=1-q \beta^{\prime} \geqslant 0$ and with Lemma 21 we get

$$
B\left(G_{n, k, b^{\prime}}\right) \lesssim\left(c_{1}(\beta+\varepsilon, k)\right) n^{k} .
$$

If $n$ sufficiently large, then $b \leqslant b^{\prime}$ and (1) implies

$$
B\left(G_{n, k, b}\right) \leqslant B\left(G_{n, k, b^{\prime}}\right) \lesssim\left(c_{1}(\beta+\varepsilon, k)\right) n^{k} .
$$

With $\varepsilon \rightarrow 0$ and the continuity of $c_{1}$ as a function of $\beta$ the assertion follows.

Now let $r=0$, i.e., $1=q \beta$. If $q=1$, then $\beta=1$. In this case, the assertion follows from Lemma 18:

$$
B\left(G_{n, k, b}\right) \leqslant\left|V_{n, k, b}\right| \sim 2 c_{2}(1, k) n^{k}=c_{1}(1, k) n^{k} .
$$

Thus let $q \geqslant 2$. For $0<\varepsilon<\frac{1}{q\left(q^{2}-q-1\right)}$ let $\beta^{\prime}=\beta+\varepsilon, b^{\prime}=\left\lfloor\beta^{\prime} n\right\rfloor$. Then $r^{\prime}=1-(q-1) \beta^{\prime} \geqslant 0$ and $\frac{(q-1)-1}{(q-1)^{2}+(q-1)-1}<r^{\prime}<\beta^{\prime}$. Note that this is Case b). Lemma 22 implies

$$
B\left(G_{n, k, b^{\prime}}\right) \lesssim\left(c_{2}(\beta+\varepsilon, k)+c_{3}(\beta+\varepsilon, k)\right) n^{k} .
$$

If $n$ is sufficiently large, then $b \leqslant b^{\prime}$ and (1) implies

$$
B\left(G_{n, k, b}\right) \leqslant B\left(G_{n, k, b^{\prime}}\right) \lesssim\left(c_{2}(\beta+\varepsilon, k)+c_{3}(\beta+\varepsilon, k)\right) n^{k} .
$$

With Lemma 23 and $\varepsilon \rightarrow 0$ we get

$$
B\left(G_{n, k, b}\right) \lesssim c_{1}(\beta, k) n^{k} .
$$


The last corollary settles Case b).

Corollary 25. Let $k \geqslant 2$ be an integer, $b \sim \beta n$ for $n \rightarrow \infty$, let $1=q \beta+r$ with a positive integer $q$ and let $\frac{q-1}{q^{2}+q-1}<r<\beta$. Then

$$
B\left(G_{n, k, b}\right) \lesssim\left(c_{2}(\beta, k)+c_{3}(\beta, k)\right) n^{k}
$$

Proof. For $0<\varepsilon<\frac{1}{q}\left(r-\frac{q-1}{q^{2}+q-1}\right)$ let $\beta^{\prime}=\beta+\varepsilon, b^{\prime}=\left\lfloor\beta^{\prime} n\right\rfloor$. Then $r^{\prime}=1-q \beta^{\prime}>\frac{q-1}{q^{2}+q-1}$ and from Lemma 22 we get

$$
B\left(G_{n, k, b^{\prime}}\right) \lesssim\left(c_{2}(\beta+\varepsilon, k)+c_{3}(\beta+\varepsilon, k)\right) n^{k}
$$

If $n$ is sufficiently large, then $b \leqslant b^{\prime}$ and (1) implies

$$
B\left(G_{n, k, b}\right) \leqslant B\left(G_{n, k, b^{\prime}}\right) \lesssim\left(c_{2}(\beta+\varepsilon, k)+c_{3}(\beta+\varepsilon, k)\right) n^{k} .
$$

With $\varepsilon \rightarrow 0$ and the continuity of $c_{2}$ and $c_{3}$ as functions of $\beta$ the assertion follows.

\section{Open problems}

We formulate the following conjecture in form of a problem because we are rather convinced that it is correct.

Problem 26. Prove that the ordering for Case b) presented in Section 10 and illustrated in Figure 7 defines an asymptotically optimal bandwidth numbering.

A larger program is formulated in the second problem:

Problem 27. Find and study other interesting graph classes that allow a reduction to the unit square for the asymptotics and lead to interesting and nontrivial orderings on the unit square.

\section{Acknowledgement}

We would like to thank the anonymous referee for valuable comments.

\section{References}

[1] R. Akhtar, T. Jiang, and Z. Miller. Asymptotic determination of edge-bandwidth of multidimensional grids and Hamming graphs. SIAM J. Discrete Math., 22(2):425449, 2008.

[2] J. Balogh, S.L. Bezrukov, L. H. Harper, and A. Seress. On the bandwidth of 3dimensional Hamming graphs. Theoretical Computer Science, 407(1-3):488-495, 2008. 
[3] T. Berger-Wolf and E. M. Reingold. Index assignment for multichannel communication under failure. IEEE Trans. Inform. Theory, 48:2656-2668, 2002.

[4] M. Blanchette, E. Kim, and A. Vetta. Clique cover on sparse networks. Proceedings of the Meeting on Algorithm Engineering 85 Experiments, ALENEX '12:93-102, 2012.

[5] P. Z. Chinn, J. Chvátalová, A. K. Dewdney, and N. E. Gibbs. The bandwidth problem for graphs and matrices: a survey. J. Graph Theory, 6:223-254, 1982.

[6] V. Chvátal. A remark on a problem of Harary. Czechoslovak Mathematical Journal, 20(1):109-111, 1970.

[7] J. Chvátalová. Optimal labelling of a product of two paths. Discrete Math., 11(3):249-253, 1975.

[8] F. R. K. Chung. Labeling of graphs. In Selected Topics in Graph Theory 3, L.W. Beineke and R.J. Wilson, eds., pages 151-168. Academic Press, 1988.

[9] E. Cuthill and J. McKee. Reducing the bandwidth of sparse symmetric matrices. Proceedings of the 1969 24th national conference, 157-172, 1969.

[10] C. Dubey, U. Feige, and W. Unger. Hardness results for approximating the bandwidth. Journal of Computer and System Sciences, 77:62-90, 2010.

[11] K. Engel and S. Hanisch. Reconstruction of cell-electrode-adjacencies on multielectrode arrays. Journal of Computational Neuroscience, 37(3):583-591, 2014.

[12] N. E. Gibbs, W. G. Poole Jr.and P. K. Stockmeyer. An algorithm for reducing the bandwidth and profile of a sparse matrix. SIAM Journal on Numerical Analysis, 13(2):236-250, 1976.

[13] S. Hanisch. Mathematische Modelle zur Rekonstruktion von Zellnetzwerken auf Multielektrodenarrays. Diss. Univ. Rostock 2017. URN: http://nbn-resolving.de/ urn:nbn:de:gbv:28-diss2017-0026-6. URL: http://rosdok. uni-rostock.de/ resolve/id/rosdok_disshab_0000001684 (accessed on March 14, 2017).

[14] L. H. Harper. Optimal numberings and isoperimetric problems on graphs. J. Combin. Theory, 1(3):385-393, 1966.

[15] L. H. Harper. On an isoperimetric problem for Hamming graphs. Discrete Appl. Math., 95:285-309, 1999.

[16] L. H. Harper. On the bandwidth of a Hamming graph. Theoretical Computer Science, 301:491-498, 2003.

[17] U. Hendrich and M. Stiebitz. On the bandwidth of graph products. J. Inform. Process. Cybernet., 28:113-125, 1992.

[18] L. T. Kou, L. J. Stockmeyer, and C. K. Wong. Covering edges by cliques with regard to keyword conflicts and intersection graphs. Communications of the ACM, 21(2):135-139, 1978.

[19] Ch. H. Papadimitriou. The NP-Completeness of the bandwidth minimization problem. Computing, 16(3):263-270, 1976. 
[20] F. Pellegrini. Bounds for the bandwidth of the $d$-ary deBruijn graph. Parallel Process. Lett., 3:431-443, 1993.

[21] S. Pissanetzky. Sparse Matrix Technology. Academic Press, London, 1984.

[22] J. S. Prentice, J. Homann, K. D. Simmons, G. Tkačik, V. Balasubramanian, and P. C. Nelson. Fast, scalable, Bayesian spike identification for multi-electrode arrays. PLoS ONE, 6(7):e19884, 2011. 